01

\title{
Асимптотически запрещенные по спину квазимолекулярные радиационные переходы (обзор)
}

\author{
(C) A.3. Девдариани ${ }^{1,2}$ \\ ${ }^{1}$ Санкт-Петербургский государственный университет, \\ 198504 Санкт-Петербург, Россия \\ ${ }^{2}$ Российский государственный педагогический университет им. А.И. Герцена, \\ 191186 Санкт-Петербург, Россия \\ e-mail: a.devdariani@spbu.ru
}

Поступила в редакцию 04.08.2021 г.

В окончательной редакции 04.08.2021 г.

Принята к публикации 10.08.2021 г.

В обзоре обсуждены теоретические работы по радиационным переходам, которые инициируются тепловыми столкновениями атомов групп 2А, 12 Таблицы Менделеева с атомами инертных газов, а также несимметричными столкновениями атомов инертных газов и приводят либо к распаду, либо к заселению нижнего метастабильного состояния ${ }^{3} P_{2}$. Обсуждение проводится в рамках аналитических подходов, основанных на использовании псевдопотенциала Ферми и полуэмпирического метода. Представлены результаты вычислений констант скоростей, коэффициентов поглощения, времен жизни колебательных состояний, формы спектров.

Ключевые слова: оптическая спектроскопия столкновения, взаимодействие квазимолекулярных состояний, псевдопотенциал Ферми, полуэмпирическая процедура восстановления термов.

DOI: $10.21883 / \mathrm{OS} .2021 .11 .51632 .2609-21$

\section{Введение. \\ Квазимолекулярные радиационные переходы. Запрещенные переходы. Запрещенные по спину переходы}

Обзор посвящен теоретическому описанию спектроскопии радиационных одноквантовых переходов в двухатомных квазимолекулах, т.е. спектров поглощения/испускания, которые формируются при медленных парных столкновениях атомов. Термин „асимптотически запрещенные“ означает как и в атомной спектроскопии, что дипольный момент такого перехода обращается в ноль, но при больших межатомных расстояниях. На первый взгляд, могло бы показаться, что нет особой необходимости уделять внимание некоторому специальному случаю сравнительно давно и хорошо разработанной физике уширения и сдвига спектральных линий, которая как раз и занимается влиянием столкновений на форму линий. Например, в известном спектроскопистам пространном обзоре [1] таким переходам, которые там названы „запрещенными“, посвящено не более одной страницы. Уже простые качественные соображения, однако, указывают на существенное различие между квазимолекулярными переходами, которые разрешены или запрещены в изолированных атомах.

Разрешенные переходы могут быть поняты и описаны, по крайней мере приближенно, как радиационные переходы между двумя квазимолекулярными состояниями, которые не взаимодействуют с другими состояниями.
В первом приближении можно считать, что центральная часть контура разрешенного квазимолекулярного перехода - лоренцева расположена по частоте вблизи соответствующего атомного перехода. Уширение контура связано с дальнодействующей частью потенциала межатомного взаимодействия, а сателлиты в далеких крыльях обусловлены возможными экстремумами в разности потенциальных кривых начального и конечного состояний.

Удовлетворительное описание запрещенных переходов в изолированных атомах строится на основе высоких порядков теории возмущения [2] и приводит к существенно меньшей по сравнению с разрешенными переходами вероятности перехода. Но ситуация кардинально меняется при наличии атомов буферного газа. Взаимодействие возбужденного атома с атомом буферного газа сопровождается понижением симметрии электронного потенциала по сравнению с изолированным атомом так, что радиационные переходы, запрещенные по четности, моменту или спину в изолированных атомах, оказываются разрешенными в квазимолекулах.

Квазимолекулярный механизм снятия запрета можно понять и описать, как правило, уже в первом порядке теории возмущения, но для нескольких состояний, взаимодействующих с исходным возбужденным состоянием в области средних межатомных расстояний $R \sim 10 a_{0}$. Разнообразие механизмов взаимодействия атомов приводит к тому, что формирующиеся полосы отличаются от лоренцевых и уже не могут быть описаны единой формулой. Другое следствие необходимости учета вза- 
имодействия возбужденных состояний заключается в том, что описание запрещенных радиационных переходов оказывается связанным с задачей неадиабатических переходов при столкновениях, так что возникает потенциальная возможность из данных по форме контура извлечь информацию о неадиабатических переходах при столкновениях атомов [3]. Обычно на первом этапе анализа запрещенных переходов решается в определенном смысле „квантово-Химическая (или статическая) задача“, т.е. определяется тем или иным способом зависимость энергетических термов и дипольных моментов квазимолекулы от межатомного расстояния, а уже затем решается задача определения формы спектра соответствующего перехода.

В настоящей работе обсуждаются результаты вычислений и проводится в ряде случаев сравнение с экспериментальными данными для переходов, которые запрещены по спину в приближении $L S$-связи в изолированных атомах. Снятие запретов по орбитальному моменту, особенно для квазимолекул атом щелочного металлаатом инертного газа, разработано достаточно подробно [3-5]. В обзорной работе по влиянию столкновений на атомные спектральные линии [1] обсуждаются только переходы $s-s, s-d$ в щелочных металлах. В коллективной монографии [6] запрещенным квазимолекулярным переходам уделено чуть более одного абзаца.

Отметим еще две тематические особенности предлагаемого обзора. В нем обсуждаются квазимолекулы двух типов, составленные из атомов групп 2А, 12 Таблицы Менделеева с возбужденной двухэлектронной конфигурацией $n s n p$ и атомов инертных газов в основных состояниях, а также несимметричные квазимолекулы, составленные из возбужденных атомов инертных газов с конфигурацией электрон-дырка $n p^{5} n^{\prime} s$ и атомов инертных газов в основных состояниях.

Такой выбор объектов сделан по двум причинам. В обоих случаях речь идет, по существу, об описании двухчастичной возбужденной конфигурации, что позволяет наиболее отчетливо проследить за механизмами снятия запретов на радиационные переходы по спину. С другой стороны, отсутствие симметрии по перестановкам атомов позволяет существенно упростить вычисления и ограничиться прозрачным аналитическим подходом для оценки взаимодействия в области средних межатомных расстояний, которая как раз и ответственна за снятие запрета на радиационный переход в квазимолекуле.

Радиационные процессы в симметричных квазимолекулах представляют отдельную и важную область тем более, что одна из первых работ по теории запрещенных переходов была выполнена Аллисоном и др. [7] по вычислению сечения излучения, индуцированного столкновениями $\mathrm{He}\left(2^{1} S\right)+\mathrm{He}\left(1^{1} S\right)$. Для статической части задачи в [7] был использован аналог ЛКАО. К радиационным процессам при симметричных столкновениях можно отнести и процессы с участием ионов, например перезарядку. В частности, резонансная перезарядка в основное состояние иона сопровождается квазимолекулярным радиационным переходом, вероятность которого обращается в ноль при больших расстояниях $R[8,9]$.

O тематике запрещенных переходов в эксперименте. По понятным причинам ее ,популярность“ существенно уступает изучению квазимолекулярных радиационных переходов, связанных с разрешенными атомными переходами. О таковых для конфигурации $n s n p$ см. обзор [10]. Тем не менее и для эксперимента обсуждаемые переходы интересны, как минимум, по двум причинам. Во-первых, их изучение по сравнению с атомными облегчается существенно большей вероятностью перехода, поскольку она обусловлена взаимодействием нескольких квазимолекулярных состояний уже в первом порядке. К тому же нет необходимости экстраполировать данные к нулевой плотности буферного газа. Во-вторых, изменение концентрации атомов в метастабильных состояниях, что зачастую определяет свойства низкотемпературной плазмы, в значительной степени обусловлено радиационными переходами, что особенно справедливо для квазимолекул, включающих тяжелый атом. Отметим, что ниже использование экспериментальных данных носит в основном иллюстративный характер и не претендует на полноту.

Ниже, если не оговорено особо, используются атомные единицы.

\section{1. Радиационные переходы $n s n p$ ${ }^{3} P_{2} \leftrightarrow n s{ }^{21} S_{0}$}

\section{1. Механизм снятия запрета}

Первое обсуждение снятия запрета на запрещенный по спину радиационный переход, индуцированный тепловыми столкновениями атомов 12 группы периодической таблицы (или по старой терминологии побочной подгруппы II группы)

$$
M\left(n s n p^{3} P_{2}\right)+X\left({ }^{1} S_{0}\right) \rightarrow M\left(n s^{21} S_{0}\right)+X\left({ }^{1} S_{0}\right)+\hbar \omega,
$$

где $M=\mathrm{Zn}, \mathrm{Cd}, \mathrm{Hg}$ с тяжелыми атомами инертных газов $X=\mathrm{Ar}, \mathrm{Kr}$, Хе было проведено в [11]. Рассмотрение было основано на вычислении матричных элементов гамильтониана квазимолекулы:

$$
\widehat{H}=\widehat{H}_{M^{*}}+\widehat{H}_{X}+\widehat{V}_{S 0}+\widehat{V},
$$

в базисе молекулярных функций, составленных из произведений соответствующих атомных в базисе $c$ по Гунду:

$$
\begin{gathered}
\left.\left|{ }^{1,3} P_{j} \Omega=1\right\rangle=\left|M\left({ }^{1,3} P_{j} m_{J}\right\rangle\right| X\left({ }^{1} S_{0}\right)\right\rangle, \\
j=1,2,3, \quad m_{j}=1 .
\end{gathered}
$$

Для одной возбужденной конфигурации матричные элементы выражаются через слэтеровский обменный интеграл $G$, коэффициент Ланде и потенциалы взаимодействия атомов $H_{\Sigma, \Pi}$ в состояниях $\Sigma$ и П без 
учета электростатического и спин-орбитального расщепления уровней атома $M(n s n p)$. В [11] эти потенциалы оценивались в рамках асимптотической теории [12,13]. Снятие запрета на радиационный переход вызывается взаимодействием трех состояний. В ходе столкновений с атомом инертного газа взаимодействие возбужденных состояний ${ }^{3} P_{1},{ }^{3} P_{2}$ приводит к расщеплению $\Sigma$ - и Птермов: $\Delta H(R)={ }^{3} H_{\Sigma}(R)-{ }^{3} H_{\Pi}(R)$, поэтому в адиабатическую квазимолекулярную волновую функцию состояния $\Omega=1^{3} P_{2}$ войдет волновая функция резонансного состояния ${ }^{1} P_{1}$, которая, в свою очередь, связана спин-орбитальным взаимодействием с функцией состояния ${ }^{3} P_{1}$ уже в изолированном атоме. Таким образом, квазистатическая радиационная ширина квазимолекулярного состояния оказывается пропорциональной вероятности спонтанного резонансного перехода ${ }^{1} P_{1} \rightarrow{ }^{1} S_{0}$. Форма спектра, усредненного по максвелловскому распределению атомов с температурой $T$, в первом приближении может быть описана как

$$
W(\Delta \omega)=\frac{\Delta \omega}{T^{2}} \exp \left(-\frac{\Delta \omega}{T}\right),
$$

где частота $\Delta \omega>0$ отсчитывается от энергии перехода ${ }^{3} P_{2} \rightarrow{ }^{1} S_{0}$. Максимумы излучения образуют переходы в окрестности точки поворота для классического движения атомов в потенциале $\Omega=1\left({ }^{3} P_{2}\right)$. Полное усредненное сечение радиационного тушения оказывается пропорциональным $T^{n}, n \approx 1.5-2.5$. Такая зависимость от температуры согласуется с экспериментальными данными, но абсолютная величина сечения для ряда пар на порядок меньше, хотя она существенно превышает оценки, основанные на теории возмущения в применении к изолированному атому. В целом, результаты [11] позволили сделать вывод о том, что при рассмотрении запрещенных переходов в атмосфере буферных газов следует привлекать квазимолекулярный механизм, но для достижения количественного согласия с экспериментом теоретический подход должен быть более аккуратным, учитывать влияние неадиабатических переходов и связанное с этим нарушение квазистатического приближения для формы спектральных линий.

\section{2. Вычисление потенцала взимодействия и радиационой ширины}

Ориентируясь на наиболее важную для рассмариваемых процессов область приложений - низкотемпературную плазму -, можно считать, что энергии столкновения атомов, образующих квазимолекулы, тепловые, поэтому для количественных оценок необходима информация о потенциалах взаимодействия при средних и больших межатомных расстояниях. Для аналитической оценки потенциалов в этой области можно воспользоваться методом эффективного гамильтониана [12]. С использованием такого подхода в [14-17] и были построены потенциалы нижних возбужденных состояний различных несимметричных квазимолекул с атомами 12 группы и инертных газов. В этом подходе гамильтониан конструируется как сумма гамильтонианов взаимодействующего возбужденного атома с атомом буферного газа без учета спин-орбитального взаимодействия:

$$
\widehat{H}=\widehat{H}_{M * X}+\widehat{V}_{S 0} .
$$

Как следует из разд. 1.1 , для расчета радиационного распада метастабильного атомного состояния ${ }^{3} P_{2}$ нужно учесть взаимодействие между квазимолекулярными состяниями с проекцией полного момента на молекулярную ось $\Omega=1$. Имея в виду последующее вычисление радиационной ширины, такой расчет удобно проводить в базисе волновых функций промежуточного между типами связей $a$ и $c$ по Гунду. Действительно, на средних межатомных расстояниях преобладает взаимодействие орбитального момента с осью, тогда как на больших расстояниях такое взаимодействие сопоставимо и меньше взаимодействия спин-орбита в возбужденном атоме. Функции промежуточного типа связи строятся в два этапа. На первом этапе из зависящих от расстояния функций $|R,(L S) \Lambda \Sigma\rangle^{(a)}$ в базисе $a$ по Гунду, которые диагонализуют гамильтониан $\widehat{H}_{M * X}$ :

$$
\begin{aligned}
\left\langle R,(L S) \Lambda^{\prime} \Sigma^{\prime}\right| & \widehat{H}_{M^{*} H}|R,(L S) \Lambda \Sigma\rangle^{(a)} \\
= & { }^{2 S+1} H_{|\Lambda|}(R) \delta_{\Lambda \Lambda^{\prime}} \delta_{\Sigma \Sigma^{\prime}},
\end{aligned}
$$

строятся функции диабатического базиса для случая $c$ :

$$
|R,(L S) j \Omega\rangle^{(c)}=\sum_{\Lambda, \Sigma}\langle L S j \Omega \mid L \Lambda S \Sigma\rangle|R,(L S) \Lambda \Sigma\rangle^{(a)},
$$

$\langle L S j \Omega / L \Lambda S \Sigma\rangle$ - коэффициенты Клебша-Гордона. В рассматриваемом случае $j=1,2, \Omega=1$, так что

$$
\begin{aligned}
|R,(10) 11\rangle^{c}=|R,(10) 10\rangle^{a}\left(=\left|{ }^{1} \Pi_{1}\right\rangle\right), & \\
|R,(11) 21\rangle^{c}= & \frac{1}{\sqrt{2}}\left[|R,(11) 10\rangle^{a}+|R,(11) 01\rangle^{a}\right] \\
& \left.\left.=\frac{1}{\sqrt{2}}\left(\left.\right|^{3} \Pi_{1}\right\rangle+\left.\right|^{3} \Sigma_{1}\right\rangle\right), \\
|R,(11) 11\rangle^{c} & =\frac{1}{\sqrt{2}}\left[|R,(11) 10\rangle^{a}-|R,(11) 01\rangle^{a}\right] \\
& \left.\left.=\frac{1}{\sqrt{2}}\left(\left.\right|^{3} \Pi_{1}\right\rangle-\left.\right|^{3} \Sigma_{1}\right\rangle\right) .
\end{aligned}
$$

В круглых скобках приведены более привычные обозначения функций случая $a$ по Гунду. Как видно из формул (8), (10), функции диабатического базиса с $j=1,2$, $\Omega=1$, но разными значеними спина не диагонализуют оператор спин-орбитального взаимодействия, хотя для атомов $\mathrm{Zn}, \mathrm{Cd}, \mathrm{Hg}$ такое взаимодействие существенно. Поэтому на втором этапе при построении волновых функций промежуточного типа связи следует перейти к линейным комбинациям:

$$
|R, j \Omega\rangle^{S}=a|R,(10) j \Omega\rangle_{L S}^{(c)}+b|R,(11) j \Omega\rangle_{L S}^{(c)}=\varphi_{1},
$$


Таблица 1. Матричные элементы $H_{i j}^{\Omega=1}$ в базисе функций типа связи $c$ для $\Omega=1$ [17]

\begin{tabular}{c|c|c|c}
\hline Базис & $\varphi_{1}$ & $\varphi_{2}$ & $\varphi_{3}$ \\
\hline \multirow{2}{*}{$H_{i j}$} & $E_{1}+a^{21} H_{\Pi}(R)+b^{2} \bar{H}(R)$ & $-(b / 2) \Delta H(R)$ & $a b\left[\bar{H}(R)-{ }^{1} H_{\text {п }}(R)\right]$ \\
& $-(b / 2) \Delta H(R)$ & $E_{2}+\bar{H}(R)$ & $-(a / 2) \Delta H(R)$ \\
& $a b\left[\bar{H}(R)-{ }^{1} H_{\Pi}(R)\right]$ & $-(a / 2) \Delta H(R)$ & $E_{3}+b^{21} H_{\Pi}(R)+a^{2} \bar{H}(R)$
\end{tabular}

$$
|R, j \Omega\rangle^{T}=-b|R,(10) j \Omega\rangle_{L S}^{(c)}+a|R,(11) j \Omega\rangle_{L S}^{(c)}=\varphi_{3},
$$

которые при больших $R$ диагонализуют гамильтониан возбужденного атома с двумя электронами вне заполненных оболочек (верхние индексы $S, T$ указывают на преобладающее значение спина). Амплитуды промежуточного типа связи $a, b$ можно выразить приближенно через константу спин-орбитального взаимодействия и кулоновский обменный интеграл [18], считая, что они слабо зависят от расстояния, либо определить из экспериментальных данных по расщеплению уровней или вероятностям переходов в атомах [19]. Третья функция $\varphi_{2}$ с $\Omega=1$ совпадает с (9). В табл. 1 приведены матричные элементы в базисе функций $\varphi_{i}(\Omega=1), E_{1}=E\left({ }^{1} P_{1}\right)$, $E_{2}=E\left({ }^{3} P_{2}\right), E_{3}=E\left({ }^{3} P_{1}\right)-$ атомные уровни энергии конфигурации $s p$,

$$
\begin{gathered}
{ }^{1,3} H_{\Pi}(R)=\left\langle{ }^{1,3} \Pi_{1}\left|\widehat{H}_{M^{*} X}\right|^{1,3} \Pi_{1}\right\rangle, \\
{ }^{3} H_{\Sigma}(R)=\left\langle{ }^{3} \Sigma_{1}\left|\widehat{H}_{M^{*} X}\right|^{3} \Sigma_{1}\right\rangle
\end{gathered}
$$

- молекулярные термы, определенные в (10) для случая $а$ без учета спин-орбитального взаимодействия, $\bar{H}(R)=\left[{ }^{3} H_{\Sigma}(R)+{ }^{3} H_{\Pi}(R)\right] / 2$. Вследствие малости ${ }^{1} H_{\Pi}$ при оценках можно считать, что ${ }^{1} H_{\Pi}={ }^{3} H_{\Pi}$ в матричных элементах $H_{13,33}$.

Диагонализация позволяет определить квазимолекулярные термы и адиабатические функции как линейные комбинации функций $(8)-(11)$ :

$$
|c, \Omega=1\rangle^{a d}=\sum_{k=1}^{3} c_{k}(R) \varphi_{k} .
$$

Для вычисления радиационных характеристик удобно вернуться в базис диабатических $c$-функций (8), (11):

$$
\begin{aligned}
& |c, \Omega=1\rangle^{a d}=\left(c_{1} a-c_{3} b\right)|R ;(10) 11\rangle_{L S}^{(c)} \\
& \quad+c_{2}|R ;(11) 21\rangle_{L S}^{(c)}+\left(c_{1} b+c_{3} a\right)|R ;(11) 11\rangle_{L S}^{(c)} .
\end{aligned}
$$

Поскольку с основным квазимолекулярным состоянием $X^{1} \Sigma^{+}$разрешенным дипольным переходом связано только синглетное состояние $|R ;(1 q 0) 111\rangle_{L S}^{(c)}$, то дипольный момент $d(R)$ радиационного перехода $c-X$ есть

$$
d(R)=\left[c_{1}(R) a-c_{3}(R) b\right]\left\langle X^{1} \Sigma^{+}|\widehat{D}| R ;(10) 11\right\rangle_{L S}^{(c)},
$$

а вероятность квазимолекулярного радиационного перехода

$$
\Gamma_{c-X}(R)=\left(\frac{a}{b} c_{1}(R)-c_{3}(R)\right)^{2}\left(\frac{\omega(R)}{\omega\left({ }^{3} P_{1}\right)}\right)^{3} \Gamma\left({ }^{3} P_{1}\right),
$$

где $\omega(R), \omega\left({ }^{3} P_{1}\right)$ - частоты квазимолекулярного и атомного переходов, $\Gamma\left({ }^{3} P_{1}\right)-$ вероятность атомного перехода.

Таким образом, для вычисления квазимолекулярных термов и вероятностей запрещенных по спину радиационных переходов необходима информация о матричных элементах ${ }^{1,3} H_{\Sigma, \Pi}$. В духе метода эффективного гамильтониана можно считать, что эти члены есть матричные элементы оператора взаимодействия атома $M^{*} \mathrm{c}$ атомом $X$ без учета спин-орбитального взаимодействия. Далее вычисления можно продолжить двумя способами, либо аналитически, например, привлекая асимптотическую теорию $[12,13]$ в сочетании с обоснованными моделями для описания взаимодействия возбужденного электрона с бесструктурным атомом $X$, либо используя полуэмпиричесую процедуру, связанную с привлечением экспериментальных данных по спектрам разрешенных квазимолекулярных переходов. Ниже будут описаны и реализованы оба подхода.

Отметим, что, видимо, первое определение по экспериментальным данным потенциальных кривых состояний, вовлеченных в асимптотически запрещенный квазимолекулярный переход, было предпринято в [20], а первое вычисление соответствующих термов в квазирелятивистском подходе $a b$ initio - в [21]. Механизм снятия запрета и определение зависимости вероятности радиационного перехода в этих работах не рассматривался.

\section{3. Квазимолекулы с легкими атомами инертных газов. Псевдопотенциал Ферми}

Основная трудность применения метода эффективного гамильтониана, введенного для рассматриваемых задач в разд. 1.2, связана с оценкой матричных элементов оператора взаимодействия атомов. Для ее преодоления предложено и реализовано два подхода. Один из них основан на использовании псевдопотенциала Ферми, первоначальная форма которого учитывала только короткодействующую часть обменного взаимодействия [22].

В рамках метода эффективного гамильтониана оператор взаимодействия сталкивающихся атомов $\widehat{H}_{M^{*} X}$ без учета спин-орбитального взаимодействия составляется как сумма гамильтонианов свободных атомов:

$$
\widehat{H}_{M^{*} X}=\widehat{H}_{M^{*}}+\widehat{H}_{X}+{ }^{2 S+1} \widehat{H}_{\Lambda}
$$

и взаимодействия ${ }^{2 S+1} \widehat{H}_{\Lambda}$, которое есть сумма:

$$
{ }^{2 S+1} \widehat{H}_{\Lambda}=\widehat{U}+\widehat{V}_{i o n}
$$


эффективного одноэлектронного оператора межатомного взаимодействия $\widehat{U}$ и оператора $\widehat{V}_{i o n}$ взаимодействия ионного остова с атомом буферного газа [23]. В области больших и средних межатомных расстояний для последнего оператора можно ограничиться приближенным выражением

$$
V_{i o n}=-\frac{\beta}{2 R^{4}},
$$

где $\beta$ - дипольная поляризуемость атома $X$.

Для вычисления матричных элементов оператора межатомного взаимодействия в случае легких инертных газов можно привлечь псевдопотенциал Ферми [22], который зависит от одного параметра $L-$ длины рассеяния возбужденного электрона на атоме буферного газа:

$$
\widehat{U}=2 \pi L \delta(\mathbf{r})+\widehat{V}^{\prime} .
$$

Оператор $\widehat{U}$ представляет сумму двух членов - короткодействующего обменного и поляризационного, учитывающего экранирование взаимодействия ионного остова $M^{+}$с атомом $X$ полем возбужденного электрона $n p$ [24]. Матричные элементы короткодействующего взаимодействия возбужденного электрона с возмущающим атомом и дальнодействующего поляризационого взаимодействия с учетом взаимодействия трех частиц $\left(M^{+}, e\right.$ и $\left.X\right)$ выполнен в работах [25-27]. Диагональные элементы для $\Lambda=\Sigma$, П приведены в [14] для синглетных и триплетных состояний:

$$
{ }^{2 S+1} I_{\Sigma}(R)={ }^{2 S+1} U_{p \Sigma}^{\mathrm{ex}}+{ }^{2 S+1} U_{p \Sigma}^{s c r},{ }^{2 S+1} H_{\Pi}(R)={ }^{2 S+!} U_{p \Pi}^{s c r} .
$$

Здесь

$$
{ }^{2 S+1} U_{p \Sigma}^{e x}=\left.\left.2 \pi^{2 S+1} B\left|Y_{10}(\mathbf{R} / R)\right|^{2}\right|^{2 S+1} f_{n p}(R)\right|^{2}
$$

— потенциал обменного взаимодействия, ${ }^{2 S+1} f(\rho)-$ радиальные волновые функции возбужденного электрона $n p$ (в качестве таковых в [14], например, использованы атомные волновые функции приближения эффективного орбитального квантового числа [28]). Длина рассеяния электрона $L$ несколько модифицируется в зависимости от области межатомных расстояний, так что

$$
{ }^{2 S+1} B=L, \text { при } R<|1|^{2 S+1} E^{*} \mid .
$$

Выражение для $U_{p \Sigma \Pi ~}^{s c r}$ приводится в [18].

\section{4. Квазимолекулы $M^{*}-\mathrm{He}, \mathrm{Ne}$. Константа скорости тушения}

Причина выделения квазимолекул, составленных из атома с двумя валентными электронами и атомом легкого инертного газа понятна из обсуждения в разд. 1.3. Для таких квазимолекул термы и радиационные ширины могут быть вычислены аналитически с использованием известных характеристик свободных атомов, в частности длины рассеяния. Для тяжелых инертных газов $X=\mathrm{Ar}$, $\mathrm{Kr}$, Хе такой подход неприемлем, поскольку значительная поляризуемость нарушает условия применимости псевдопотенциала Ферми [25]. Другое ограничение, но уже связанное с возбужденным атомом, заставляет в этом разделе ограничиться $M=\mathrm{Cd}, \mathrm{Ba}, \mathrm{Yb}, \mathrm{Hg}$. Причина в том, что для легких атомов расстояние между вовлеченными в процесс уровнями ${ }^{3} P_{1,2}$ оказывается сопоставимым с тепловой энергией столкновения, так что при анализе радиационных процессов необходимо учитывать и недиабатические переходы (подробнее в разд. 1.8). Учитывая эти ограничения, целесообразно начать обсуждение с квазимолекулы $\mathrm{Hg}^{*}-\mathrm{He}$, тем более что малость потенциальных ям в основном и возбужденном состояниях затрудняет использование методов лазерно индуцированной флуоресценции. Именно этими методами получены наиболее надежные данные по характеристикам излучающих состояний, которые необходимы в случае тяжелых инертных газов (см. ниже разд. 1.5). В случае $\mathrm{Hg}^{*}-\mathrm{He}$ экспериментальные спектроскопические характеристики получены только для состояния $0^{+}\left({ }^{3} P_{1}\right)$ [29]. Результаты теоретических работ, в основе которых лежат соображения, изложенные в разд. 1.2, 1.3, уже излагались в обзорных работах [30,31], поэтому ниже обсуждаются только некоторые новые результаты [32].

К подходам разд. 1.2, 1.3 есть два связанных вопроса насколько оправдано использованное одноконфигурационное приближение и возможно ли представление взаимодействия ионной оболочки с нейтральным атомом простым поляризационным потенциалом (18). Ответы на эти вопросы позволяют уточнить и области межатомных расстояний, для которых, тем не менее, оказывается возможным применение упрощенного подхода.

Для изучения влияния межконфигурационного взаимодействия целесообразно воспользоваться аналитическими выражениями для матричных элементов взаимодействия, полученными в $[26,33,34]$. Впервые такой подход к изучению влияния межконфигурационного взаимодействия был применен в [35] для квазимолекулы $\mathrm{Li}^{*}-$ Не. Сравнение с многоконфигурациоными расчетами ab initio [35] продемонстрировало хорошее согласие результатов, что позволило перенести подход [34], сочетающий аналитические оценки взаимодействия и учет многоконфигурационного взаимодействия, и на квазимолекулу $\mathrm{Hg}^{*}-\mathrm{He}$.

В таком подходе в [23] показано, что для состояний ${ }^{1,3} П$ влияние взаимодействия с ближайшими 13 возбужденными конфигурациями $n s, d, f$ при $R \leq 7 a_{0}$ несущественно. Для конфигураций ${ }^{1,3} \Sigma$ влияние взаимодействия конфигураций оказывается более существенным, но для тепловых столкновений такое взаимодействие лишь незначительно меняет положение точки поворота на классической траектории в области $R \sim(8-9) a_{0}$. В целом, результаты многоконфигурационных расчетов $[34,35]$ позволяют сделать вывод о том, что даже одноконфигурационное приближение дает удовлетворительные результаты при энергиях связи электрона в атоме менее $5 \mathrm{eV}$ и оправдано для атомов 


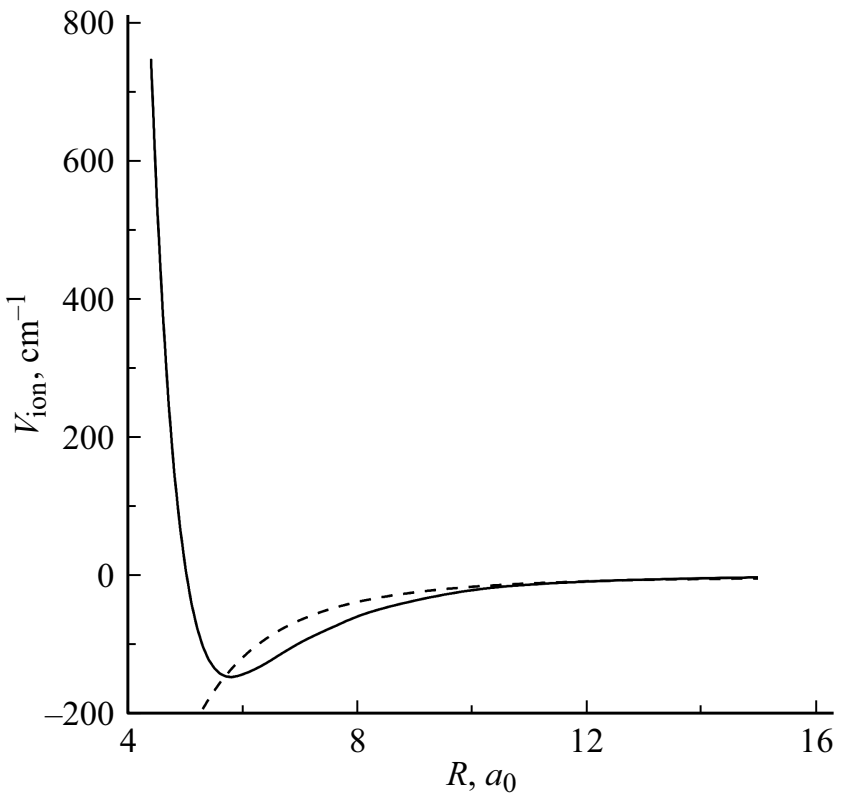

Рис. 1. Потенциальная кривая ион-атомного взаимодействия $\mathrm{Hg}^{+}-\mathrm{He}$. Сплошная кривая - результат неэмпирического расчета $V_{\text {ion }}$, штриховая кривая - поляризационное взаимодействие $V_{\text {ion-p }}=-\beta / 2 R^{4}$.

Таблица 2. Параметры потенциалов взаимодействия для $\mathrm{Hg}\left(6^{1,3} P_{J}\right)+\mathrm{He}$

\begin{tabular}{c|c|c|c|c|c|c}
\hline Состояние & \multicolumn{3}{|c|}{$R_{e}, a_{0}$} & \multicolumn{3}{|c}{$D_{e}, \mathrm{~cm}^{-1}$} \\
\hline Варианты расчетов & $a$ & $b$ & $c$ & $a$ & $b$ & $c$ \\
\hline $0^{-}\left({ }^{3} P_{0}\right)$ & 8.7 & 10.0 & 12 & 13 & 3.0 & 1.6 \\
$0^{+}\left({ }^{3} P_{1}\right)^{d}$ & 6.2 & - & 11.5 & 58 & - & 1.9 \\
$1\left({ }^{3} P_{1}\right)$ & 9.1 & 10.4 & 12.3 & 10.4 & 3.1 & 1.3 \\
$2\left({ }^{3} P_{2}\right)$ & 5.8 & - & 7.0 & 108 & - & 29 \\
$1\left({ }^{3} P_{2}\right)$ & 9.1 & 10.5 & 12.4 & 10 & 3.0 & 1.1 \\
$0^{-}\left({ }^{3} P_{2}\right)$ & 9.5 & 10.7 & $>13.5$ & 8 & 3.1 & 0.8 \\
$1\left({ }^{1} P_{1}\right)$ & 5.8 & - & 7.4 & 131 & - & 20 \\
$0^{+}\left({ }^{1} P_{1}\right)$ & 14.0 & 14.4 & $>14$ & 1.3 & 1.3 & -
\end{tabular}

Примечание. $a$ - результаты вычислений [32]; $b$ - вычисления [23] с использованием поляризационного приближения для потенциала $V_{\text {ion }}$; $c$ - вычисления [34] с использованием потенциала из [42] для состояний ${ }^{1,3} \Sigma$ и ${ }^{1,3} \Pi ; d-$ для состояния $0^{+}\left({ }^{3} P_{1}\right)$ экспериментальные значения $R_{e}, D_{e}$ и $D_{0}$ равны соответственно $R_{e}=6.54$ [43], 6.6 [44], 6.8 $a_{0}$ [45]; $D_{e}=28 \mathrm{~cm}^{-1}$ [43]; $D_{0}=28$ [44], $29 \pm 2$ [45], $13 \pm 2 \mathrm{~cm}^{-1}[43]$.

с малой поляризуемостью, т. е. для Не, Ne. Менее обнадеживающие результаты получены в [31] для описания взаимодействия ионного остова с атомом буферного газа $V_{\text {ion. }}$ Использование простой формулы (18) в случае $X=$ Не оправдано только для $R \geq 7 a_{0}$, где обменное отталкивание иона $\mathrm{Hg}^{+}$и атома $\mathrm{He}$ несущественно. В области меньших расстояний следует использовать потенциал ион-атомного взаимодействия, который получен каким-либо независимым методом, например, из экспериментальных данных или в результате неэмпири- ческого расчета. Именно так был получен потенциал ион-атомного взаимодействия $\mathrm{Hg}^{+}-\mathrm{He}$ в [32] (рис. 1) путем использования многоотсылочного метода конфигурационного взаимодействия с учетом однократных и двукратных возбуждений (MRD-CI) [36-40] в сочетании с методом релятивистского эффективного остовного потенциала (RECP) [39-41]. Как видно из рис. 1, при $R<5.5 a_{0}$ использование формулы (18) неприемлемо. Корректный учет отталкивательной части ионатомного потенциала позволил определить термы в более широкой по сравнению с [23] областью расстояний (рис. 2), а также характеристики наиболее глубоких потенциальных ям состояний $1\left({ }^{1} P_{1}\right), 2\left({ }^{3} P_{2}\right), 0^{+}\left({ }^{3} P_{1}\right)$ (табл. 2, там же приводятся экспериментальные данные для состояния $0^{+}$). Отметим, что вычисленное значение вдвое превышает экспериментальное, тогда как расчет с использованием матричных элементов из работы [42] (подробнее в разд. 1.5 ) занижает величину $D_{e}$ более чем на порядок.

Согласно картине термов (рис. 2), обусловленный квазимолекулярным радиационным переходом $1\left(6^{3} P_{2}\right)-0^{+}\left(6^{1} S_{0}\right)$ распад метастабильного состояния $\mathrm{Hg}\left(6^{3} P_{2}\right)$ формирует полосу, которая сдвинута в корот-

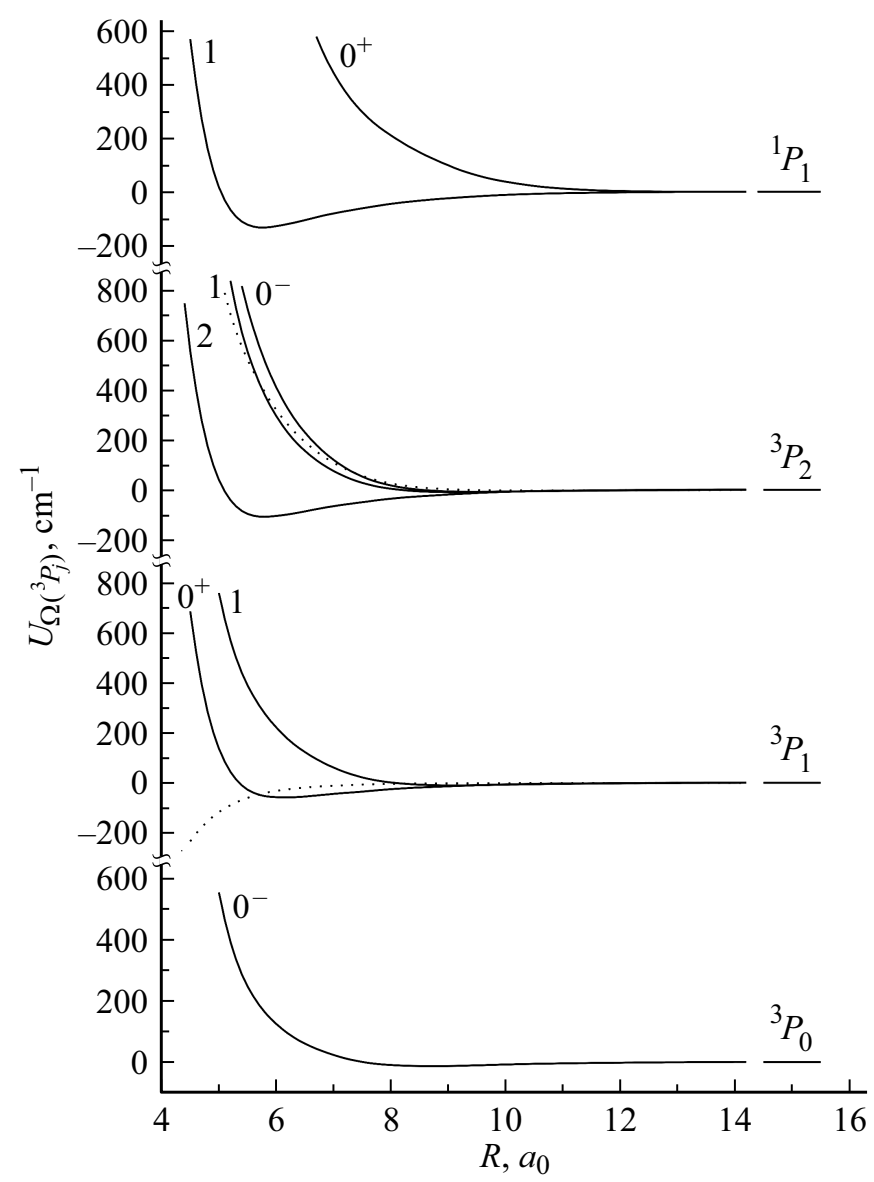

Рис. 2. Потенциалы взаимодействия $\mathrm{Hg}(6 s 6 p)-$ Не. Сплошные кривые - расчет [32], штриховые кривые - расчет [23] для состояний $1\left({ }^{3} P_{2}\right)$ и $0^{+}\left({ }^{3} P_{1}\right)$ с учетом только поляризационной составляюей ион-атомного взаимодействия. 
Таблица 3. Константы скорости $K_{c-X}$ (в $\left.10^{-18} \mathrm{~cm}^{3} \mathrm{~s}^{-1}\right)$ столкновительно индуцированного радиационного распада $\mathrm{Hg}\left(6 s 6 p^{3} P_{2}\right)+\mathrm{He}\left({ }^{1} S_{0}\right) \rightarrow \operatorname{Hg}\left(6 s^{21} S_{0}\right)+\operatorname{He}\left({ }^{1} S_{0}\right)+\hbar \omega$

\begin{tabular}{c|c|c|c}
\hline$T, \mathrm{~K}$ & \multicolumn{3}{|c}{$K_{c-X}$} \\
\hline Варианты рачетов & $a$ & $b$ & $c$ \\
\hline 100 & 0.41 & 0.26 & 0.26 \\
200 & 1.0 & 0.87 & 0.82 \\
300 & 1.8 & 1.7 & 1.6 \\
400 & 2.6 & 2.8 & 2.6 \\
500 & 3.5 & 4.0 & 3.8 \\
600 & 4.4 & 5.2 & 5.1 \\
700 & 5.3 & 6.5 & 6.4 \\
800 & 6.2 & 7.9 & 7.8 \\
900 & 7.1 & 9.3 & 9.2 \\
1000 & 7.9 & 10.6 & 10.7
\end{tabular}

Примечание. $a$ - результаты вычислений [32]; $b$ - вычисления [15] с учетом вклада только поляризационного взаимодействия $V_{\text {ion }-p}$ при многоконфигурационном расчете термов; $c$ - с учетом вклада только поляризационного взаимодействия $V_{\text {ion }-p}$ при одноконфигурационном расчете термов.

коволновую область по отношению к частоте запрещенного атомного перехода $\omega_{2}\left(6^{3} P_{2}-6^{1} S_{0}\right)$. Величина сдвига $\hbar \Delta \omega_{m}$ максимума полосы излучения относительно $\omega_{2}$, а также ширина распределения сопоставимы с $k T$.

Практический интерес представляет константа скорости радиационного тушения:

$$
K(T)=\frac{2}{5} 4 \pi \int_{0}^{\infty} \Gamma(R) \exp [-U(R) k T] R^{2} d R
$$

в процессе

$$
\operatorname{Hg}\left(6 s 6 p^{3} P_{2}\right)+\operatorname{He}\left({ }^{1} S_{0}\right) \rightarrow \operatorname{Hg}\left(6 s^{21} S_{0}\right)+\operatorname{He}\left({ }^{1} S_{0}\right)+\hbar \omega,
$$

где $\Gamma, U-$ радиационная ширина и потенциал взаимодействия состояния $c 1\left({ }^{3} P_{2}\right), 2 / 5-$ статистический множитель. Результаты расчета $K(T)$ в различных приближениях в интервале изменения температуры от 100 до $1000 \mathrm{~K}$ приведены в табл. 3. Увеличение константы с температурой связано с уменьшением расстояния наибольшего сближения атомов и сопутствующего увеличения обменного взаимодействия. Отметим, что учет взаимодействия конфигураций оказывает слабое влияние на величину $K(T)$ по сравнению с влиянием поляризационного ион-атомного взаимодействия $[32,46]$.

В одноконфигурационном приближении были выполнены также и расчеты для атомов $\mathrm{Yb}, \mathrm{Ba}, \mathrm{Cd}$ (включение лантаноида $\mathrm{Yb}$ оправдано, так как многие уровни $\mathrm{Yb}^{*}$ могут быть рассмотрены в двухэлектронном прибижении) [47]. Как и в случае атома ртути, квазимолекулярное излучение формирует узкий пик в окрестности запрещенного атомного перехода ${ }^{3} P_{2}-{ }^{1} S_{0}$ шириной $1000 \mathrm{~cm}^{-1}$. Значения соответствующих констант скоростей приведены в табл. 4. Вычисления проводились по формуле (22), которая, строго говоря, справедлива только для потенциала отталкивания $U(R)$, но в случае $X=\mathrm{He}, \mathrm{Ne}$ потенциальные ямы $D_{e} \ll k T$, поэтому ограничением, связанным с центробежным барьером, можно пренебречь (подробнее см. разд. 2.5). Особенность для вычисленных значений $K(T)$ состоит в том, что они близки к соответствующим величинам для случая $\mathrm{Hg}^{*}$ - Не. Связано это с тем, что уменьшение отклонения от $L S$-связи по сравнению с атомом $\mathrm{Hg}$ и сопутствующее уменьшение вероятности радиационного атомного перехода ${ }^{3} P_{1}-{ }^{1} S_{0}$ компенсируется сдвигом диапазона сильного смешивания функций состояний $1\left({ }^{3} P_{1},{ }^{3} P_{2}\right)$ при уменьшении $\Delta=E_{2}-E_{3}$ в область бо́льших расстояний.

Достоинство изложенного выше подхода, который сочетает методы эффективного гамильтониана и псевдопотенцала, в том, что он сравнительно легко переносится на случай молекулярных буферных газов, например, $\mathrm{Hg}^{*}-\mathrm{H}_{2}$. Квадрупольное взаимодействие возбужденного электрона с молекулой смешивает состояния с различными проекциями электронного углового момента на ось $z$, которая соединяет возбужденный атом и центр масс молекулы $\mathrm{H}_{2}$, и приводит к зависимости энергии состояний от угла $\theta$ между осью $z$ и осью молекулы. На рис. 3 в качестве примера показана зависимость взаимодействия $\mathrm{Hg}\left(6^{3} P_{0}\right)-\mathrm{H}_{2}$ от $R$ и $\theta$ [31]. В этом расчете не учитывалась зависимость обменного взаимодействия ионного остова с молекулой водорода от $R$ и $\theta$, так что результаты справедливы только в области $R>6 a_{0}$, тем не менее использованный подход и расчет можно было бы использовать для анализа крыльев спектральных линий, порожденных столкновениями с молекулами.

Таблица 4. Константы скорости $\left(K \times 10^{18}, \mathrm{~cm}^{3} \mathrm{~s}^{-1}\right)$ столкновительно индуцированного радиационного распада $M\left({ }^{3} P_{2}\right)+X\left({ }^{1} S_{0}\right) \rightarrow M\left({ }^{1} S_{0}\right)+X\left({ }^{1} S_{0}\right)+\hbar \omega ; \quad M=\mathrm{Cd}, \mathrm{Ba}, \mathrm{Hg}$, $\mathrm{Yb} ; X=\mathrm{He}, \mathrm{Ar}, \mathrm{Kr}, \mathrm{Xe}$

\begin{tabular}{c|c|c|c|c|c|c|c}
\hline \multirow{2}{*}{$T, \mathrm{~K}$} & \multicolumn{7}{|c}{$M X$} \\
\cline { 2 - 8 } & $\mathrm{CdHe}$ & $\mathrm{BaHe}$ & $\mathrm{YbHe}$ & $\mathrm{HgNe}^{*}$ & $\mathrm{HgAr}^{*}$ & $\mathrm{HgKr}^{*}$ & $\mathrm{HgXe}^{*}$ \\
\hline 100 & 0.07 & 0.24 & 0.29 & 0.33 & 1.8 & 5.0 & 13.4 \\
200 & 0.20 & 0.62 & 0.89 & 0.86 & 2.9 & 6.6 & 15.5 \\
300 & 0.36 & 1.00 & 1.61 & 1.5 & 4.3 & $8.5^{* *}$ & $18.5^{* * *}$ \\
400 & 0.53 & 1.37 & 2.40 & 2.3 & 5.7 & 10.5 & 20.5 \\
500 & 0.68 & 1.74 & 3.25 & 3.1 & 7.2 & 12.5 & 23.0 \\
600 & 0.87 & 2.12 & 4.12 & 4.0 & 8.6 & 14.6 & 25.9 \\
700 & 1.05 & 2.50 & 5.15 & 4.9 & 10.3 & 16.7 & 28.7 \\
800 & 1.22 & 2.90 & 6.27 & 5.9 & 11.9 & 18.8 & 31.5 \\
900 & 1.39 & 3.20 & 7.55 & 8.1 & 13.5 & 21.0 & 34.4 \\
1000 & 1.55 & 3.86 & 9.04 & 7.9 & 15.1 & 23.2 & 37.3
\end{tabular}

Примечание. * - вычисления [46]; ** - экспериментальные значения (в единицах $10^{-18} \mathrm{~cm}^{3} \mathrm{~s}^{-1}$ ) при $T=305,346$ и $373 \mathrm{~K}$ равны соответственно 6.1, 6.9 и 7.5 [9]; *** — экспериментальные значения (в единицах $\left.10^{-18} \mathrm{~cm}^{3} \mathrm{~s}^{-1}\right)$ при $T=303,330$ и $385 \mathrm{~K}$ равны соответственно $130 \pm 22,140 \pm 30$ и $170 \pm 20$ [48] и $K(293 \mathrm{~K})=16$ [49]. 


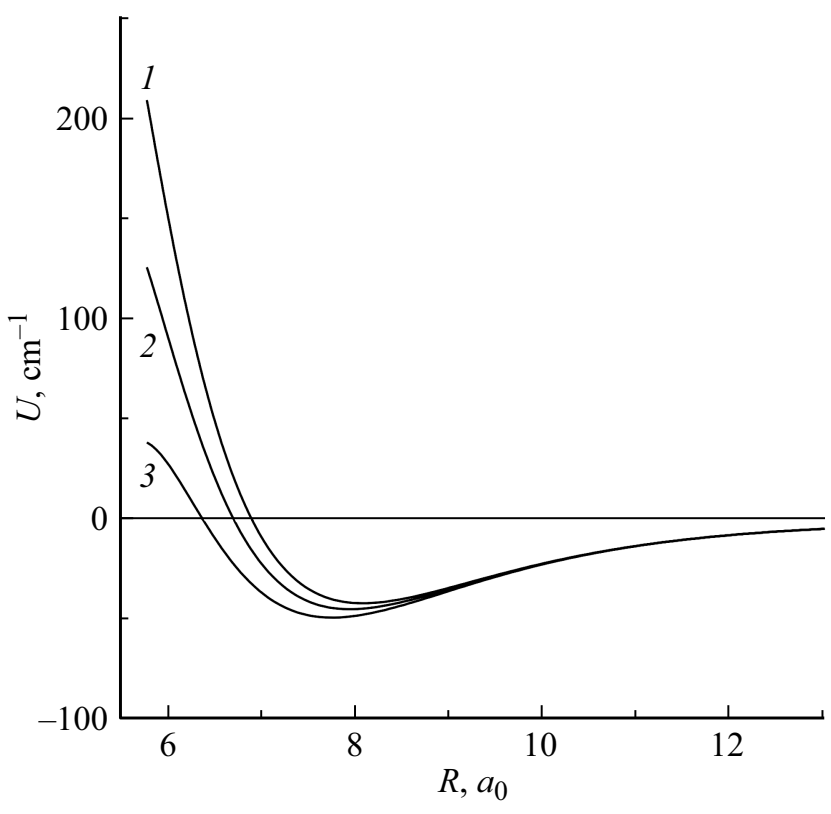

Рис. 3. Потенциалы взаимодействия $\mathrm{Hg}(6 s 6 p)-\mathrm{H}_{2}$ при $\theta=0(1), \pi / 4(2), \pi / 2(3)$.

\section{5. Квазимолекулы $M^{*}+\mathrm{Ar}, \mathrm{Kr}$, Хе. Константа скорости тушения}

Сильное поляризационное взаимодействие возбужденного электрона с тяжелым атомом инертного газа препятствует использованию псевдопотенциала Ферми. Тем не менее, предложенный в разд. 1.2 подход, основанный на вычислении матричных элементов эффективного гамильтониана, позволяет получить реалистичные оценки потенциалов и вероятностей радиационных переходов вовлеченных квазимолекулярных состояний. Оценка основана на том, что зависимости от расстояния всех восьми квазимолекулярных состояний, порожденных конфигурацией $n s n p$, могут быть выражены через четыре функции ${ }^{1,3} H_{\Sigma, \Pi}(R)-$ потенциалы взаимодействия в состояниях $\Sigma$ и П без учета спин-орбитального расщепления.

Действительно, матричные элементы для состояний с $\Omega=1$ (табл. 1) уже использовались в разд. 1.2 , а для состояний с $\Omega=0^{+}$они приведены в табл. 5 . Диагонализация этих матриц позволяет определить, в частности, термы А $0^{+}\left({ }^{3} P_{1}\right)$ и В $1\left({ }^{3} P_{1}\right)$ (рис. 4$)$, которые участвуют в формировании крыльев разрешенного перехода ${ }^{3} P_{1}-{ }^{1} S_{0}$, красного и фиолетового соответственно. Сопоставляя полученные термы с теми, которые могут быть определены из экспериментальных данных по крыльям спектральной линии, можно восстановить зависимости матричных элементов ${ }^{1,3} H_{\Sigma, \Pi}(R)$, если принять, что синглетные и триплетные элементы одинаковы. Оценка погрешности такого упрощения в [16] показала, что замена не приводит к существенному изменению потенциалов.
Таблица 5. Матричные элементы $H_{i j}^{\Omega=1}$ в базисе функций типа связи $c$ для $\Omega=0^{+}[17]$

\begin{tabular}{c|c|c}
\hline Базис & $\left|R, D, 10^{+}\right\rangle^{S}$ & $\left|R, A, 10^{+}\right\rangle^{T}$ \\
\hline$H_{i j}$ & $E_{1}+a^{21} H_{\Sigma}(R)+b^{23} H_{\text {п }}(R)$ & $-a b \Delta H(R)$ \\
& $-a b \Delta H(R)$ & $E_{3}+b^{21} H_{\Sigma}(R)+a^{23} H_{\Pi}(R)$
\end{tabular}

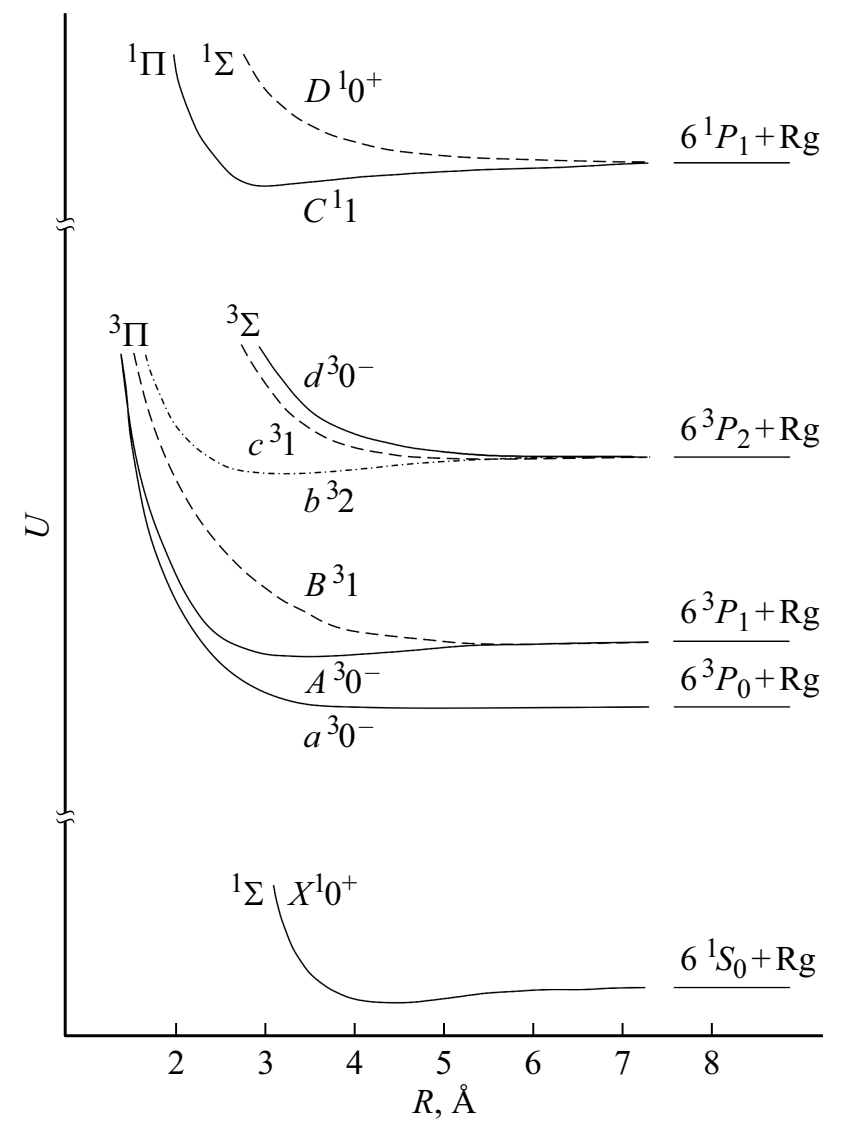

Рис. 4. Схематическая картина квазимолекулярных термов $M^{*}(n s n p)-\mathrm{Ar}, \mathrm{Kr}, \mathrm{Xe}$.

В табл. 6, составленной по данным [16,51], приводятся характеристики потенциальных кривых состояния $1\left({ }^{3} P_{2}\right)$ для ряда квазимолекул, восстановленных в рамках полуэмпирического подхода, а также полученные непосредственно в эксперименте [50]. Восстановленные потенциалы справедливы при $R>7 a_{0}$, а их общая характерная черта - наличие ямы, способной поддержать несколько колебательных состояний. Здесь уместно сделать одно общее замечание по поводу полуэмпирического метода определения термов. Дело в том, что определенные по экспериментальным данным термы $0^{+}\left({ }^{3} P_{1}\right), 1\left({ }^{3} P_{1}\right)$, которые используются в виде потенциалов Морзе для восстановления термов конфигурации $n s n p$, строго говоря, справедливы в окрестности минимумов излучающих состояний. В этой же области межатомных расстояний должны быть справедливы и 
Таблица 6. Характеристики потенциальных ям состояния с $\Omega=1\left({ }^{3} P_{2}\right)$ квазимолекул $\operatorname{Hg}\left(6^{3} P_{2}\right)[16], \mathrm{Cd}\left(6^{3} P_{2}\right)[51]-\mathrm{Ne}$, $\mathrm{Ar}, \mathrm{Kr}, \mathrm{Xe}$

\begin{tabular}{c|c|c|c}
\hline Квазимолекула & $R_{e}, a_{0}$ & $D_{e}, \mathrm{~cm}^{-1}$ & $\omega, \mathrm{cm}^{-1}$ \\
\hline $\mathrm{Hg}-\mathrm{Ne}$ & 9.34 & 13 & 8.0 \\
$\mathrm{Hg}-\mathrm{Ar}$ & 9.00 & 51 & 12.5 \\
& $8.88^{*}$ & $61.1^{*}$ & $11^{* *}$ \\
& & $56^{* *}$ & \\
$\mathrm{Hg}-\mathrm{Kr}$ & 8.70 & 91 & \\
& $8.75^{*}$ & $103.3^{*}$ & \\
$\mathrm{Hg}-\mathrm{Xe}$ & 8.83 & 180 & 12.5 \\
$\mathrm{Cd}-\mathrm{Ar}$ & 9.48 & 57 & 12 \\
$\mathrm{Cd}-\mathrm{Kr}$ & 9.25 & 108 & 11
\end{tabular}

Примечание. * - по данным [17], ** - по данным [50].

восстановленные термы. Поэтому зависимость термов от расстояния и связанная с этим надежность описания далеких крыльев спектральных линий, формируемых переходами вдали от области минимумов излучающих потенциалов, не могут претендовать на большую точность.

Поскольку диагонализация части гамильтониана для состояний $\Omega=1$ (табл. 1) определяет и коэффициенты $c_{i}$ в формуле (13), то описанная процедура восстановления потенциалов позволяет определить и радиационную ширину состояния с $1\left({ }^{3} P_{2}\right)$. Удобно ввести безразмерную приведенную радиационную ширину

$$
\gamma_{c-X}(R)=\frac{\Gamma_{c-X}(R)}{\Gamma\left({ }^{3}\left(P_{1}\right)\right.}\left(\frac{\Omega\left({ }^{3} P_{1}\right)}{\Omega_{c-X}(R)}\right)^{3},
$$

которая не зависит от основного состояния квазимолекулы и характеризует отношение квадратов дипольного момента квазимолекулы и дипольного момента атомного перехода ${ }^{3} P_{1}-{ }^{1} S_{0}$. Как пример, на рис. 5 приведены графики $\gamma(R)_{c-X}$ из работы [52] квазимолекул $\mathrm{Hg}^{*}-\mathrm{He}, \mathrm{Ne}$, $\mathrm{Ar}, \mathrm{Kr}, \mathrm{Xe}$. Для радиационных ширин характерна сильная зависимость от расстояния, а также обращение в нуль в окрестности $R \sim 9-10 a_{0}$. Последнее обстоятельство связано с тем, что в используемом приближении дипольный момент пропорционален $\Delta H=H_{\Sigma}-H_{П}$, поэтому при больших $R$, где преобладает дальнодействующее взаимодействие, $\Delta H<0$, а при малых $R$ преобладает обменное взаимодействие и $\Delta H>0$.

Одно замечание перед обсуждением результатов по константам скоростей радиационного тушения метастабильного состояния $M^{*}\left({ }^{3} P_{2}\right)$. Выше уже отмечалось существование потенциальных ям в терме $c 1\left({ }^{3} P_{2}\right)$, сопоставимых с $k T$, что приводит к ограничению области параметров удара при классическом движении атомов. Это ограничение следует учитывать, когда речь идет об излучении, индуцированном парными свободно-свободными и свободно-связанными переходами при столкновениях, поскольку частично в эту область дают вклад и переходы из связанных состояний в потенциальной яме

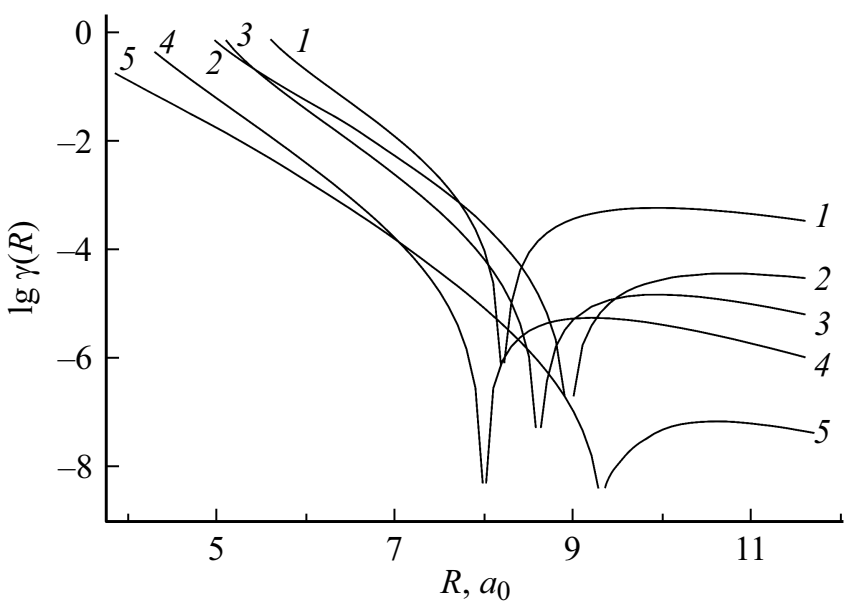

Рис. 5. Приведенные радиационные ширины $\gamma\left(c 1\left({ }^{3} P_{2}\right)\right)$ в зависимости от $R$ : $\mathrm{Hg}-\mathrm{Xe}(1), \mathrm{Hg}-\mathrm{Kr}(2 \mid /), \mathrm{Hg}-\mathrm{Ar}(3), \mathrm{Hg}-\mathrm{Ne}(4)$, $\mathrm{Hg}-\mathrm{He}(5)$ [52].

Таблица 7. Константы скорости $K_{c-X} \quad\left(\right.$ в $\left.10^{-17} \mathrm{~cm}^{3} \mathrm{~s}^{-1}\right)$ столкновительно индуцированного радиационного распада $\mathrm{Hg}\left(6 s 6 p^{3} P_{2}\right)$ в $\mathrm{Ne}, \mathrm{Ar}, \mathrm{Kr}, \mathrm{Xe}$

\begin{tabular}{c|c|c|c|c}
\hline$T, \mathrm{~K}$ & $K_{c-X}(\mathrm{Ne})$ & $K_{c-X}(\mathrm{Ar})$ & $K_{c-X}(\mathrm{Kr})$ & $K_{c-X}(\mathrm{Xe})$ \\
\hline 100 & 0.033 & 0.18 & 0.64 & 1.34 \\
200 & 0.086 & 0.29 & 0.89 & 1.55 \\
300 & 0.15 & 0.43 & 1.13 & 1.85 \\
400 & 0.23 & 0.57 & 1.35 & 2.05 \\
500 & 0.31 & 0.72 & 1.58 & 2.30 \\
600 & 0.40 & 0.86 & 1.79 & 2.59 \\
700 & 0.49 & 1.03 & 2.01 & 2.87 \\
800 & 0.59 & 1.19 & 2.22 & 3.15 \\
900 & 0.81 & 1.35 & 3.43 & 3.44 \\
1000 & 0.79 & 1.51 & 2.65 & 3.73
\end{tabular}

возбужденного состояния [53-55]. В табл. 7 приведены результаты вычисления констант скоростей тушения для квазимолекул $\mathrm{Hg}^{*}-\mathrm{Ne}, \mathrm{Ar}, \mathrm{Kr}$, Хе по формуле (22), которая учитывает оба типа переходов как из свободных, так и из связанных состояний. В работе [15] приводятся также и результаты вычислений константы тушения, которые учитывают переходы только с участием свободных атомов. Учитывая незначительную в масштабе $k T$ глубину потенциальной ямы, результаты вычислений в двух предельных случаях совпадают даже для пары $\mathrm{Hg}^{*}-\mathrm{Xе} \mathrm{при} T>500 \mathrm{~K}$. Для $X=\mathrm{He}, \mathrm{Ne}$ можно считать, что взаимодействие носит отталкивательный характер.

Для квазимолекул с атомом Cd условие, при котором можно пренебречь недиабатическими переходами из состояния ${ }^{3} P_{2}$ в ближайшее атомное излучающее состояние ${ }^{3} P_{1}$, выполняется несколько хуже по сравнению со случаем атома $\mathrm{Hg}$, расщепление равно $1171 \mathrm{~cm}^{-1}$ против $4631 \mathrm{~cm}^{-1}$ в атоме $\mathrm{Hg}$. Потенциальные кривые и радиационные ширины для квазимолекул $\mathrm{Cd}-\mathrm{Ar}, \mathrm{Kr}$, вычисленные в обсуждаемом выше подходе, приводятся 
Таблица 8. Вероятности $A\left(v^{\prime}, v^{\prime \prime}\right)$ (в $\left.\mathrm{s}^{-1}\right)$ переходов $v^{\prime} 1\left({ }^{3} P_{2}\right)-v^{\prime \prime} 0^{+}\left({ }^{1} S_{0}\right)$ в квазимолекулах $\mathrm{Hg}-\mathrm{Ar}, \mathrm{Kr}, \mathrm{Xe}$

\begin{tabular}{|c|c|c|c|c|c|c|}
\hline $\mathrm{Rg}$ & \multicolumn{6}{|c|}{$\mathrm{Ar}$} \\
\hline \multirow[t]{2}{*}{$v^{\prime \prime}$} & \multicolumn{6}{|c|}{$v^{\prime}$} \\
\hline & 0 & 1 & 2 & 3 & 4 & 5 \\
\hline 0 & 88 & 277 & 500 & 673 & - & - \\
\hline 1 & 409 & 900 & 1157 & 1143 & - & - \\
\hline 2 & 555 & 635 & 365 & 123 & - & - \\
\hline 3 & 197 & 23 & 41 & 171 & - & - \\
\hline 4 & 0 & 38 & 97 & 51 & - & - \\
\hline 5 & 17 & 2 & 0 & 26 & - & - \\
\hline $\mathrm{Rg}$ & \multicolumn{6}{|c|}{$\mathrm{Kr}$} \\
\hline \multirow[t]{2}{*}{$v^{\prime \prime}$} & \multicolumn{6}{|c|}{$v^{\prime}$} \\
\hline & 0 & 1 & 2 & 3 & 4 & 5 \\
\hline 0 & 16 & 77 & 210 & 410 & 660 & 940 \\
\hline 1 & 190 & 700 & 1470 & 2320 & 3040 & 3520 \\
\hline 2 & 820 & 2140 & 3150 & 3400 & 3010 & 2290 \\
\hline 3 & 1670 & 2460 & 1780 & 700 & 80 & 44 \\
\hline 4 & 1560 & 670 & 0.1 & 433 & 1080 & 1290 \\
\hline 5 & 540 & 44 & 730 & 770 & 280 & 4 \\
\hline $\mathrm{Rg}$ & \multicolumn{6}{|c|}{$\mathrm{Xe}$} \\
\hline$v^{\prime \prime}$ & \multicolumn{6}{|c|}{$v^{\prime}$} \\
\hline & 0 & 1 & 2 & 3 & 4 & 5 \\
\hline 0 & 140 & 669 & 1765 & 3418 & 5545 & 7577 \\
\hline 1 & 910 & 3090 & 5690 & 7520 & 7920 & 6970 \\
\hline 2 & 2190 & 4500 & 4400 & 2410 & 530 & 23 \\
\hline 3 & 2380 & 1950 & 190 & 440 & 2130 & 3240 \\
\hline 4 & 1040 & 18 & 920 & 1850 & 1040 & 73 \\
\hline
\end{tabular}

Таблица 9. Радиационные времена $\tau\left(v^{\prime}\right)$ состояний $v^{\prime} c 1\left({ }^{3} P_{2}\right)$ квазимолекул $\mathrm{CdAr}$ и $\mathrm{CdKr}[51,64]$

\begin{tabular}{c|c|c}
\hline \multirow{2}{*}{$v^{\prime}$} & \multicolumn{2}{|c}{$\tau\left(v^{\prime}\right), \mathrm{s}$} \\
\cline { 2 - 3 } & $\mathrm{CdAr}$ & $\mathrm{CdKr}$ \\
\hline 0 & $3.7 \cdot 10^{-3}$ & $1.3 \cdot 10^{-3}$ \\
1 & $1.6 \cdot 10^{-3}$ & $6.2 \cdot 10^{-4}$ \\
2 & $1.2 \cdot 10^{-3}$ & $4.3 \cdot 10^{-4}$
\end{tabular}

в работах [56,57]. Как и в случае $\mathrm{Hg}$, для потенциальной кривой $c 1\left({ }^{3} P_{2}\right)$ характерно наличие потенциальной ямы при $R \sim 9 a_{0}$ глубиной $\sim 100 \mathrm{~cm}^{-1}$, а для ширины наличие корня в этой же области расстояний.

Выше, за исключением обсуждения квазимолекулы $\mathrm{Hg}-\mathrm{H}_{2}$ в разд. 1.4, предполагалось, что буферный газ $X-$ одноатомный, бесструктурный. Переход к двухатомным молекулам, даже без учета электронного возбуждения, усложняет анализ квазимолекулярных оптических переходов. В работе [57] впервые было измерено поглощение в далеком крыле столкновительного комплекса $\operatorname{Hg}\left({ }^{1} S_{0} \rightarrow{ }^{3} P_{2}\right)-\mathrm{N}_{2}$, CO, которое сопро- вождалось не только преимущественным заселением состояния $\operatorname{Hg}\left({ }^{3} P_{2}\right)$, но и заселением состояния $\operatorname{Hg}\left({ }^{3} P_{1}\right)$, что, разумеется, отсутствует при замене молекулярного буфера инертным газом. Этот результат объяснялся в [57] возбуждением колебательных состояний молекул $\mathrm{N}_{2} \mathrm{~K}, \mathrm{CO}$, которое позволяет уменьшить энергетический дефект между начальными и конечными состояниями.

Несколько замечаний в заключение этого раздела. Представленные результаты были основаны на развитии полуэмпирического метода вычисления термов и радиационных ширин. По существу, такой подход продолжает изложенный в [58] полуэмпирический подход к анализу атомных термов, в рамках которого термы одной конфигурации выражаются через несколько параметров, которые, в свою очередь, определяются при сопоставлении с экспериментальными данными для излучающих состояний. Другой важный момент - построение атомных волновых функций промежуточного типа связи в базисе функций $L S$-связи, что можно было бы назвать базисом с-функциями нулевого порядка [15-17,59].

Обратим также внимание на то, что дипольный момент оптического перехода меняет знак, т.е. меняет ориентацию на противоположную, приблизительно на границе области доминирования дальнодействующих или обменных сил. Здесь ситуация также напоминает известное в физике атомного фотоэффекта обращение в ноль дипольного момента [60,61], но при определенной частоте поглощения. Наличие нулей дипольного момента в зависимости от расстояния между ядрами с зарядами $Z_{1,2}$ установлено и при точном вычислении моментов в одноэлектронной задаче [62].

\section{6. Времена жизни колебательных состояний в потенциале $c 1\left({ }^{3} P_{2}\right)$}

Результаты расчетов, приведенные в разд. 1.5, предсказывают наличие потенциальных ям $D_{e} \sim 100 \mathrm{~cm}^{-1}$ при $R_{e} \sim 9 a_{0}$ для квазимолекул $\mathrm{Hg}, \mathrm{Cd}-\mathrm{Ar}, \mathrm{Kr}, \mathrm{Xe} . \mathrm{B}$ [50] сообщено о первом прямом экспериментальном наблюдении связанно-связанного перехода $c-X$. Оптическое возбуждение колебательных состояний в потенциале $c 1\left({ }^{3} P_{2}\right)$ для $\mathrm{Hg}-\mathrm{Ar}$ контролировалось последующим возбуждением в вышележащее состояние $E$. Было определено положение 7 колебательных уровней, что позволило построить график Бирджа-Спонера и определить $D_{e}=56 \mathrm{~cm}^{-1}$ и $\omega_{r}=11 \mathrm{~cm}^{-1}$ (аппроксимация потенциалом Морзе) в согласии с данными табл. 6.

Следующий вопрос, который связан с колебательными состояниями и может представить интерес для физики лазерных сред - оценка вероятностей радиационных переходов $v^{\prime}$, которая, согласно разд. 1.2 , (17) определяется как

$$
A\left(v^{\prime}, v^{\prime \prime}\right)=\frac{\omega\left({ }^{3} P_{)} 2\right)}{\omega\left({ }^{3} P_{1}\right)} \Gamma\left({ }^{3} P_{1}\right)\left|\left\langle v^{\prime}\left|\frac{a c_{1}}{b}-c_{3}\right| v^{\prime \prime}\right\rangle\right|^{2},
$$

где $\left|v^{\prime}, v^{\prime \prime}\right\rangle$ - волновые функции колебательных состояний в возбужденном терме $c 1\left({ }^{3} P_{2}\right)$ и основном терме 


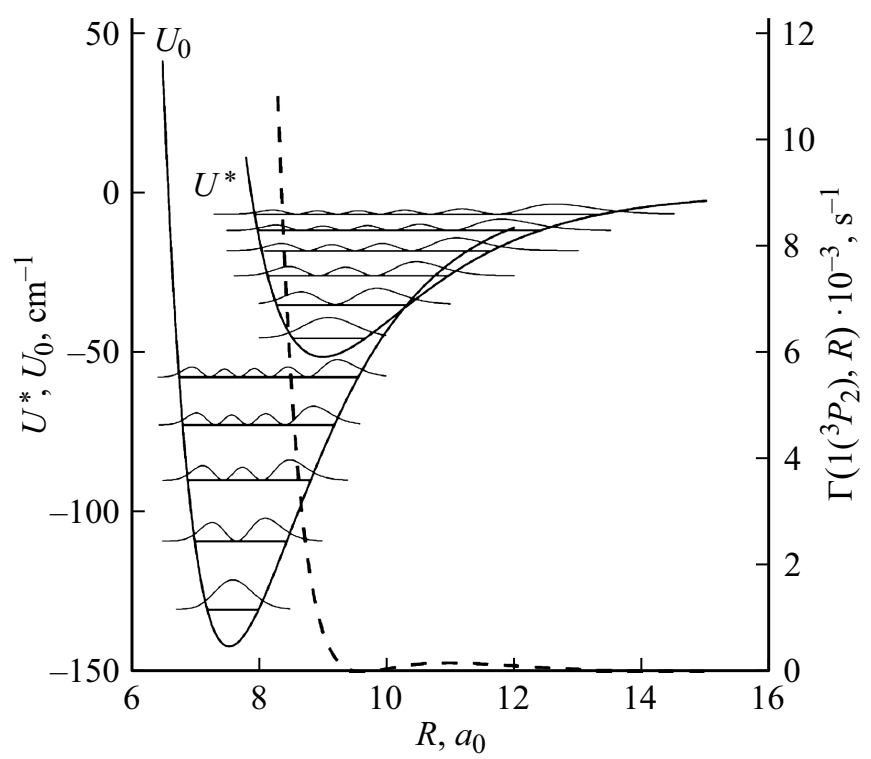

Рис. 6. Квазимолекула CdKr. Потенциалы Морзе для возбужденного, $U^{*}\left(c 1\left({ }^{3} P_{2}\right)\right)$, и основного, $U_{0}$, состояний, положения уровней энергии колебательных состояний и распределения плотности вероятности (сплошные линии), вероятность квазимолекулярных радиационных переходов (штриховая линия) [65].

$X\left({ }^{1} S_{0}\right)$, а также оценка времени жизни состояния

$$
\tau^{-1}\left(v^{\prime}\right)=\left\langle v^{\prime}\left|\Gamma\left(1\left({ }^{3} P_{2}\right), R\right)\right| v^{\prime}\right\rangle .
$$

Расчеты обеих характеристик выполнены в работе [63] для квазимолекулы $\mathrm{Hg}-\mathrm{Rg}$ и в работе $[51,64]$ для $\mathrm{Cd}-\mathrm{Rg}$. В этих работах для потенциалов основных состояний использовались данные работ [65] для $\mathrm{Hg}$ и [66] для $\mathrm{Cd}$. В качестве примера в табл. 8 приводятся данные по вероятностям переходов для квазимолекул с атомом $\mathrm{Hg}$, а в табл. 9 - данные по временам жизни для квазимолекул с атомом $\mathrm{Cd}$. Здесь уместно упомянуть, что время жизни атомных метастабильных состояний равны $\tau_{m}=6.5 \mathrm{~s}\left(\mathrm{Hg}\left(6^{3} P_{2}\right)\right), 130 \mathrm{~s}\left(\mathrm{Cd}\left(5^{3} P_{2}\right)\right)$ [67].

В целом, вероятности переходов изменяются в широких пределах, что обусловлено влиянием сильной зависимости дипольного момента перехода от межатомного расстояния и степенью перекрытия колебательных функций начального и конечного состояний (рис. 6) [64]. Для нижних уровней $\left(v^{\prime}<3\right)$ уменьшение времени жизни с увеличением $v^{\prime}$ обусловлено именно резким возрастанием радиационной ширины с уменьшением межатомного расстояния. Дальнейшее увеличение колебательного возбуждения сопровождается уменьшением перекрытия функций и смещением перехода в область средних расстояний, где ширина менятся уже незначительно, так что верхние колебательные состояния остаются долгоживущими. Тем не менее отметим, что взаимодействие, например, с атомом $\mathrm{Kr}$ уменьшает время жизни эксимера $\mathrm{Hg}, \mathrm{Cd}-\mathrm{Kr}$ в $10^{4}-10^{5}$ раз по сравнению с изолированным атомным состоянием ${ }^{3} P_{2}$.

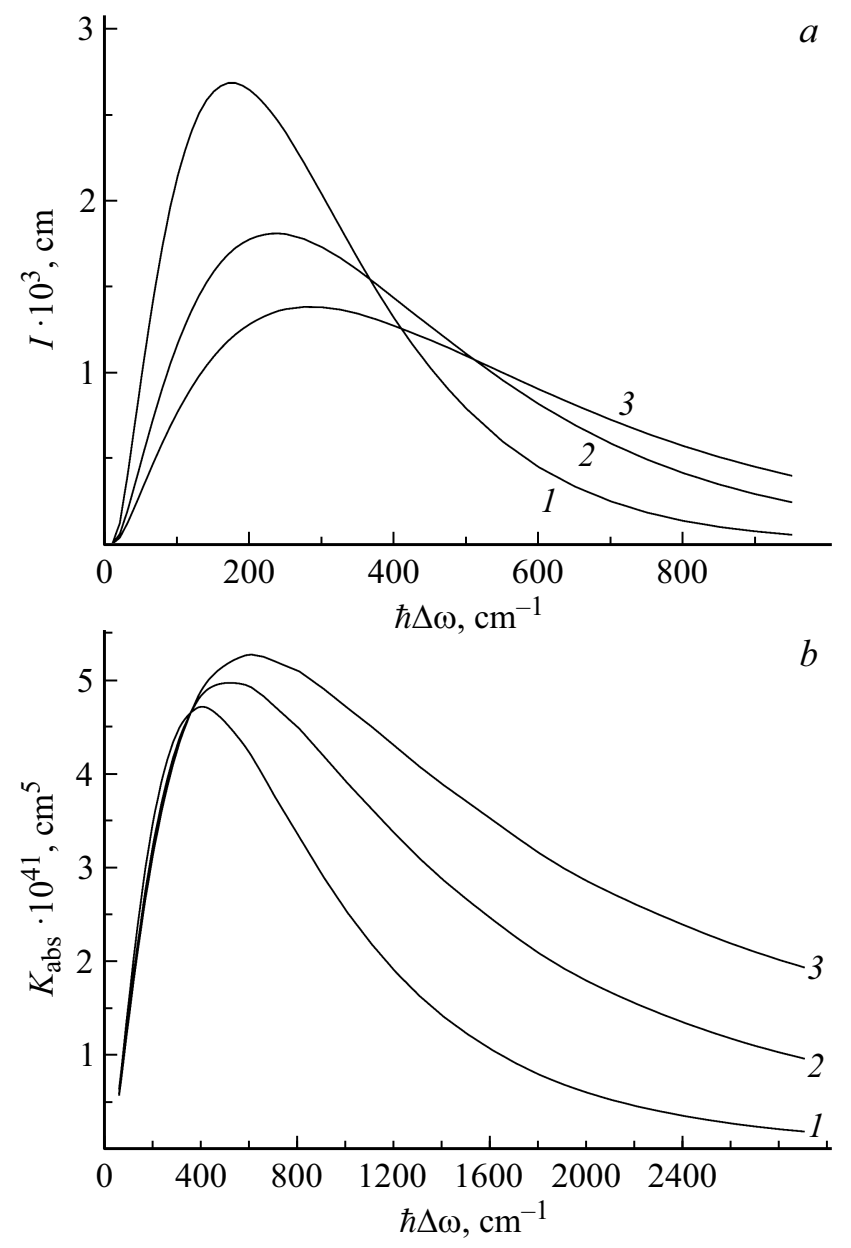

Рис. 7. Нормированные спектральные распределения квазимолекулярного излучения $(a)$ и поглощения $(b)$ $\mathrm{Hg}\left(6^{3} P_{2}\right)-\mathrm{Ne}[75]: T=300(1), 500$ (2), $700 \mathrm{~K}(3)$.

\section{7. Спектр радиационных переходов с участием состояния $c 1\left({ }^{3} P_{2}\right)$ В квазистатическом приближении}

Ниже рассматриваются формы спектров поглощения/излучения для квазимолекул $\mathrm{Hg}, \mathrm{Cd}-\mathrm{Ar}, \mathrm{Kr}, \mathrm{Xe}$. Для указанных пар имеющиеся экспериментальные данные по формам спектров асимптотически разрешенных переходов позволяют, используя полуэмпирическую процедуру, восстановить потенциальную кривую состояния $c 1\left({ }^{3} P_{2}\right)$ и радиационную ширину. Другая особенность выбранных квазимолекул - возможность пренебречь при тепловых столкновениях неадиабатическими переходами между состояниями, которые при больших $R$ вырождаются в атомные ${ }^{1} P_{1},{ }^{3} P_{2}$. В этом случае радиационный переход можно описать как переход между двумя состояниями с энергиями $U_{0}+\hbar \omega$ и $U^{*}\left(c 1\left({ }^{3} P_{2}\right)\right)$.

Принципиальные основы квантового описания радиационных процессов при столкновениях атомов при таких условиях заложены в основном в работах [68-71], о дальнейшем развитии см. [72]. В этом подходе, называемом квазистатической теорией, предполагается, 

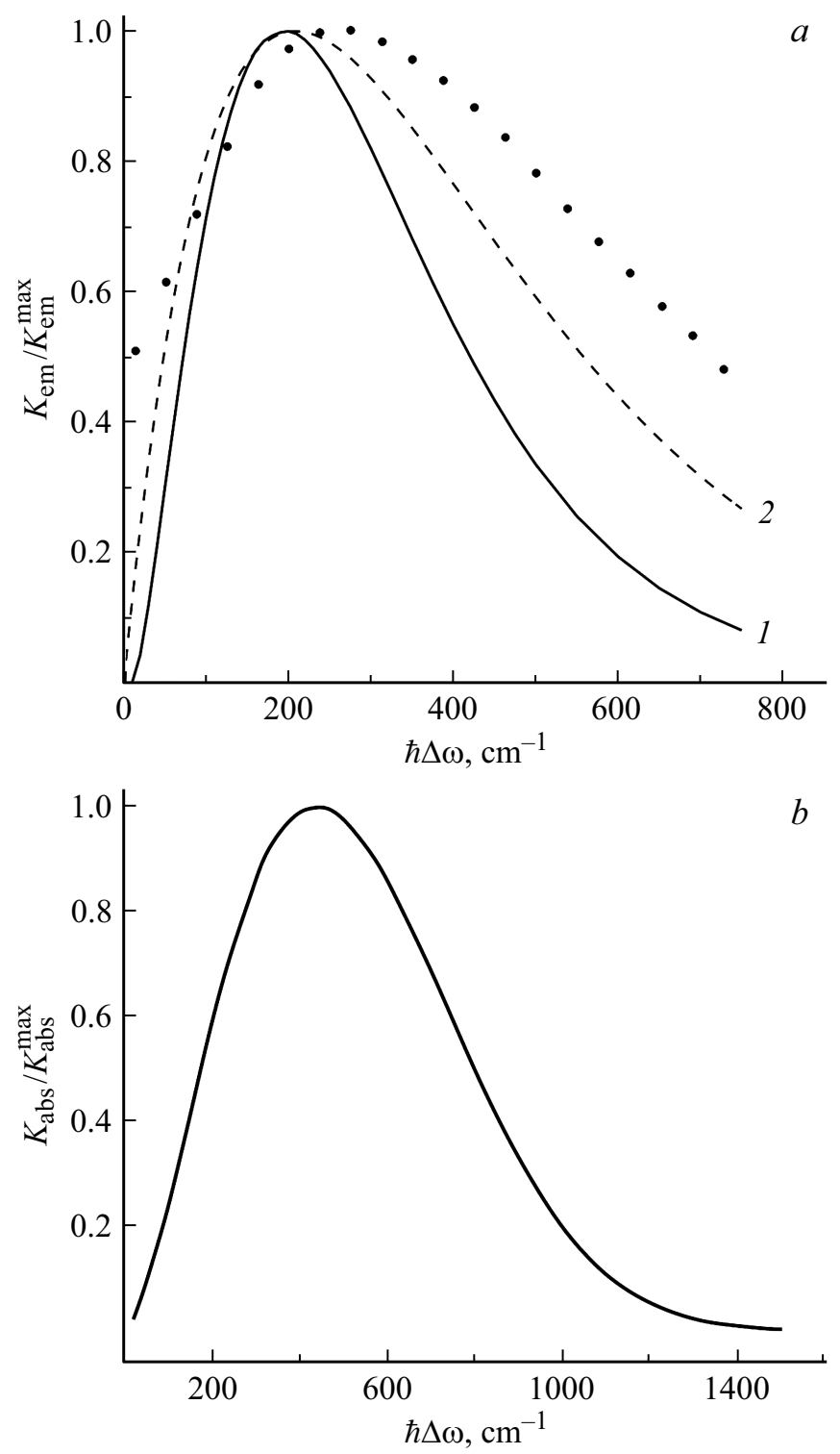

Рис. 8. Квазимолекулярные радиационные переходы $\mathrm{Hg}\left(6^{3} P_{2}\right)-\mathrm{Ar}: \quad(a)$ излучение при $T=292 \mathrm{~K}$, отнесенное к максимальному в полосе, точки - эксперимент [59], 1 расчет [75], 2 - асимптотическая оценка [4]; (b) поглощение, также отнесенное к максимальному [17], в масштабе графика экспериментальные и расчетные данные совпадают.

что радиационный переход между двумя электронными состояниями в квазимолекуле происходит в окрестности кондоновской точки $R_{c}$, определяемой условием равенства энергии фотона и разностного потенциала, т. е.

$$
\Delta U\left(R=R_{c}\right)=U^{*}-U_{0}=\hbar \omega .
$$

Привлечение полуклассического приближения для траектории атомов позволяет написать простую формулу для формы спектра. Оказывается [73], что как и в случае изолированного атома, достаточно вычислить фурьекомпоненту от матричного элемента дипольного момента $D$, но который вычисляется теперь на зависящих от времени волновых функциях квазимолекулы. То есть, например, амплитуда излучения фотона имеет вид

$$
b(\omega)=-i \int \sqrt{\frac{2\left(\alpha \omega^{3}\right)}{3 \pi g}} D(t) \exp \left[-i \int[\Delta U-\omega] d t^{\prime}\right] d t,
$$

где квадрат предэкспоненциального множителя есть с точностью до $2 \pi$ коэффициент Эйнштейна (или радиационная ширина Г) для излучения квазимолекулы в момент времени $t, \alpha-$ постоянная тонкой структуры. После суммирования по параметрам удара и усреднению по максвелловскому распределению скоростей формула (27) приводит к известному выражению для крыла спектральной линии в излучении в квазистатическом приближении:

$$
K(\omega, T)=4 \pi R_{c}^{2} \frac{\Gamma\left(R_{c}\right)}{\left|\frac{d}{d R} \Delta U\left(R_{c}\right)\right|} \exp \left[-\frac{U^{*}\left(R_{c}\right)}{k T}\right]
$$

и к коэффициенту поглощения

$$
\gamma(\omega, T)=\pi \lambda^{2} R_{c}^{2} \frac{\Gamma\left(R_{c}\right)}{\left|\frac{d}{d R} \Delta U\left(R_{c}\right)\right|} \exp \left[-\frac{U_{0}\left(R_{c}\right)}{k T}\right] .
$$

Последние две формулы после умножения (28) на $2 / 5$ использовались для вычисления коротковолновых крыльев спектров квазимолекулярных переходов $c-X$ при столкновениях атомов $\mathrm{Hg}, \mathrm{Cd}$ с атомами инертных газов в основном состоянии $[74,75]$. В расчете использовались термы и радиационные ширины, полученные с использованием метода певдопотенциала разд. 1.4, а также в случае $\mathrm{Ne}$ с использованием полуэмпирического метода. Подробное обсуждение термов и сравнение неэмпирических результатов с полуэмпирическими для этих пар выполнено в [14,32], а результаты для контуров излучения и поглощения для различных температур газовой ячейки в случае $\mathrm{Hg}-\mathrm{Ne}[75]$ приведены на рис. 7. Их отличают две особенности, которые обусловлены характером зависимости термов и радиационной ширины от межатомного расстояния. Максимум поглощения сдвинут в коротковолновую область по сравнению с излучением, поскольку отталкивание возбужденного электрона атомами Не, Ne проявляется при больших расстояниях по сравнению с отталкиванием нормальной оболочкой $n s^{2}$, так что переходы сосредоточены в области, где радиационная ширина мала. По этой же причине отсутствуют и переходы в длинноволновой области спектра $\omega<\omega_{0} \quad\left({ }^{3} P_{2} \leftrightarrow{ }^{1} S_{0}\right)$. Другая особенность заметный рост поглощения с повышением температуры, поскольку ее рост приводит к уменьшению расстояния наибольшего сближения атомов и увеличению вероятности перехода. Возможное влияние переходов из связанных и квазисвязанных состояний обсуждается ниже.

Остановимся теперь на переходах в квазимолекулах $\mathrm{Hg}-\mathrm{Rg}, \mathrm{Rg}=\mathrm{Ar}, \mathrm{Kr}, \mathrm{Xe}$. Для случая $\mathrm{Ar}$ вычисления для излучения и поглощения выполнены в [75], а температурная зависимость формы спектра излучения вычислена и сопоставлена с эксперментальными данными в [65] 
(рис. 8,a). В [17] поглощение контролировалось последующим переходом $6^{3} P_{2} \rightarrow 7^{3} S_{1}$, а экспериментальные результаты сопоставлялись с расчетами в подходе, описанном в разд. 1.2, 1.3, с использованием экспериментальных данных по переходам $\mathrm{Hg}\left({ }^{3} P_{1}-{ }^{1} S_{0}\right)-\operatorname{Ar}[76]$ (рис. $8, b$ ). И расчеты, и экспериментальные данные не только для $\mathrm{Hg}-\mathrm{Ar}$, но для $\mathrm{Hg}-\mathrm{Kr}, \mathrm{Xe}$ [77] подтверждают основные выводы, полученные из рассмотрения квазимолекул $\mathrm{Hg}-\mathrm{He}, \mathrm{Ne}$. Квазимолекулярные переходы при парных столкновениях формируют сателлит, расположенный в коротковолновой области по отношению к атомному переходу $\mathrm{Hg}\left({ }^{3} P_{2}-{ }^{1} S_{0}\right)$, положение максимума которого сдвигается в сторону больших частот с увеличением температуры. Различие в положениях максимума поглощения и излучения связано с характером зависимости термов основного и возбужденного состояний и радиационной ширины от расстояния (рис. 9). Поскольку эти зависимости были построены в $[16,52]$ на основе различных экспериментальных работ, то рис. 9 позволяет судить и об „устойчивости“ полуэмпирического метода определения термов и ширины.

Для квазимолекул с атомами тяжелых инертных газов $X=\mathrm{Ar}, \mathrm{Kr}, \mathrm{Xe}$ характерно наличие минимума в терме $c 1\left({ }^{3} P_{2}\right)$ при $R \sim 10 a_{0}$ глубиной $D_{e} \sim k T$ (табл. 6). Поэтому в отличие от $X=\mathrm{He}, \mathrm{Ne}$ встает вопрос, как влияют на форму спектра переходы с участием колебательных состояний $v, v^{\prime}$ возбужденного и основного состояний. С учетом таких состояний вместо (1) следовало бы записать процессы излучения и поглощения в условиях газовой ячейки как

$$
\begin{aligned}
M\left({ }^{3} P_{2}\right) & +\operatorname{Rg}, M \operatorname{Rg}\left(c 1^{1} P_{2}, v\right) \leftrightarrow M\left({ }^{1} S_{0}\right) \\
& +\operatorname{Rg}+\hbar \omega, M \operatorname{Rg}\left(X^{1} S_{0}, v\right)+\hbar \omega .
\end{aligned}
$$

В приближении парных столкновений и для классических траекторий этот вопрос при вычислении формы спектра на основе формулы (28) решается посредством ограничения верхней границы области интегрирования по параметрам удара значением параметра орбитирования $[53,55]$. Для экспериментов в условиях газовой ячейки полученный таким образом контур в излучении соответствует пределу низкой плотности. В пределе высокой плотности конверсия с увеличением плотности инертного газа и трехчастичные столкновения приводят к термодинамически равновесному заселению колебательных состояний в терме $c 1\left({ }^{3} P_{2}\right)$. Так что в этом пределе, когда

$$
\kappa \tau_{p}[N]>1
$$

( - константа скорости колебательной релаксации, $\tau_{p}$ - характерное радиационное время жизни состояния, $[N]$ - плотность атомов), вновь оказывается справедливой формула (28) [54]. Как показано в [46], орбитирование приводит к уменьшению доли переходов в излучении с незначительным сдвигом от положения запрещенного атомного перехода. Влияние орбитирования на форму спектра излучения $\mathrm{Hg}-\mathrm{Kr}$, Хе учтено
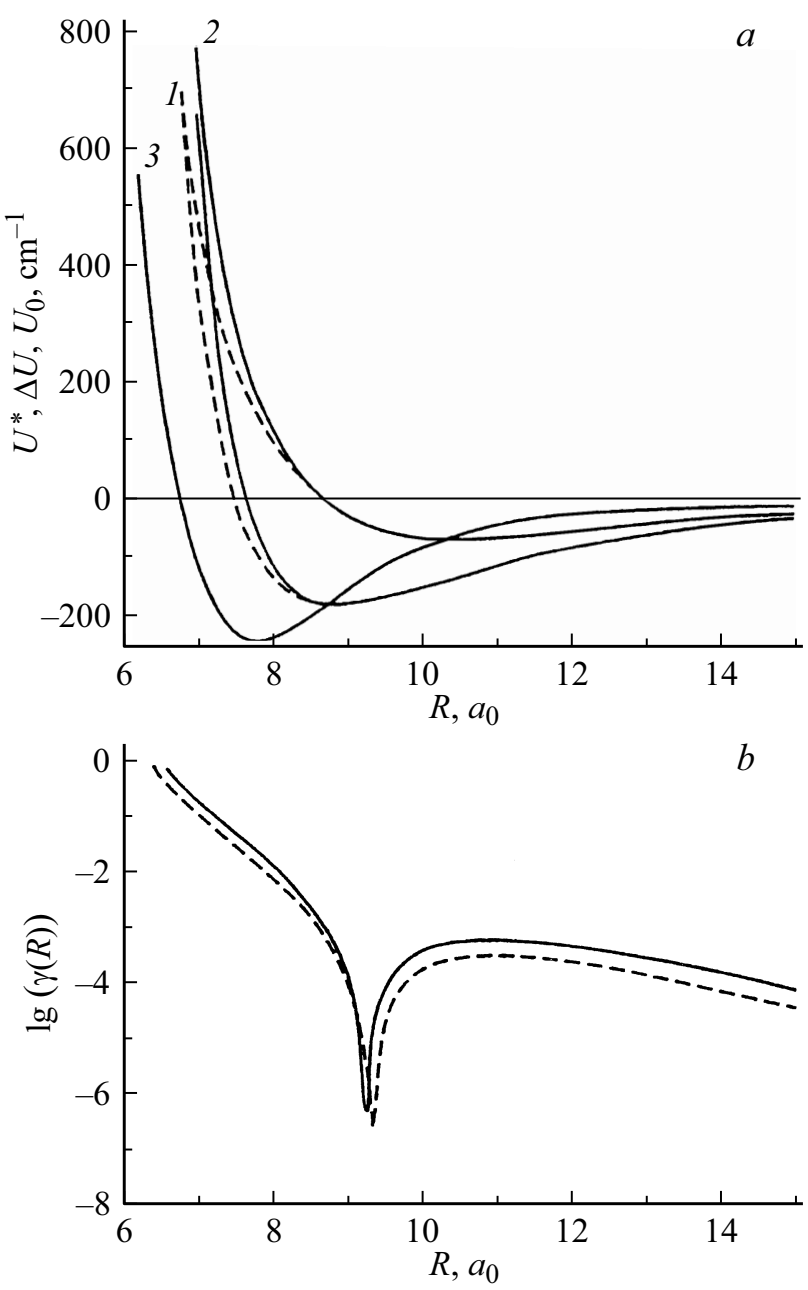

Рис. 9. (a) Потенциалы взаимодействия Нg-Хе: 1 $U^{*}\left(c 1\left({ }^{3} P_{2}\right), 2\right.$ - разностный потенциал $\Delta U, 3$ - потенциал основного состояния $U_{0} ;(b)$ радиационная ширина, отнесенная к ширине атомного состояния Хе $\left(5 p 6 s^{3} P_{2}\right)$. Сплошные и штриховые линии соответствуют двум разным наборам экспериментальных данных для излучающих квазимолекулярных состояний [77].

в [77]. На поглощение влияние орбитирования, видимо, не рассматривалось, хотя в этом случае эффект должен быть более значительным, учитывая бо́льшую глубину потенциальной ямы в основном состоянии. Влияние отклонения от термодинамического равновесия на профиль крыла спектральной линии рассматривалось в [78]. Как следует из результатов этой работы в условиях экспериментов в газовой ячейке по излучению колебательных состояний в терме $c 1\left({ }^{3} P_{2}\right)$, можно считать, что возбужденные молекулы находятся в термодинамически равновесном состоянии.

Как отмечалось в разд. 1.5, для квазимолекул $\mathrm{Cd}-\mathrm{Rg}$ представление о состоянии $c 1\left({ }^{3} P_{2}\right)$, не связанном неадиабатическим взаимодействием с другими состояниями, уже не столь оправдано. Поэтому в [54] спектральные профили, индуцированные столкновениями $\mathrm{Cd}\left(5^{3} P_{2}\right)$ с атомами $\mathrm{Ar}, \mathrm{Kr}$, вычислены для $T=300$ и $700 \mathrm{~K}$. Как и в 
случае $\mathrm{Hg}$, спектр в излучении представляет непрерывную полосу, сдвинутую в коротковолновую область по сравнению с положением запрещенной атомной линии $\mathrm{Cd}\left(5^{1} S_{0}-5^{3} P_{2}\right)$. Поскольку глубина потенциальной ямы начального состояния меньше $k T$ (табл. 6), то полоса формируется в основном переходами при двухчастичных столкновениях в окрестности точки поворота при классическом движении атомов, причем положение максимума излучения слабо зависит от температуры, а его величина уменьшается с повышением температуры. В поглощении также формируется непрерывная полоса в коротковолновой области, что приводит к селективному заселению состояния $5^{3} P_{2}$ атомов с кинетической энергией, превышающей тепловую.

\section{8. Влияние неадиабатических переходов на форму спектра квазимолекул}

В предыдущем разделе спектры вычислялись с помощью формул квазистатического приближения (28), (29), которые очень наглядны - это просто вероятности испускания/поглощения фотона парой атомов на расстоянии $R$. Получение таких формул основано на оценке интеграла (27) по методу перевала. Недостатки формул связаны с тем, что они не учитывают возможность существования экстремума в разностном потенциале $\Delta U(t)$, который, например, обязательно имеет место в окрестности точки поворота классической траектории и приводит к появлению „радуги“ [79]. В работе [80] получена формула, которая учитывает интерференционные эффекты при наличии экстремума в разностном потенциале, если экстремум расположен вдали от точки поворота классической траектории атомов. Привлечение квадратичной аппроксимации $\Delta U(t)$ в окрестности кондоновской точки позволило выразить в $[79,80]$ амплитуду перехода через функцию Эйри. Недостаток использованного приближения очевиден, оно справедливо только для переходов в окрестности экстремума. Более общее описание, основанное на аппроксимации $\Delta U(t)$ функций Морзе и экспоненциальной зависимости радиационной ширины, предложено в $[81,82]$. В этом приближении амплитуда выражается через функцию параболического цилиндра с комплексным показателем, что позволило единым образом описать лоренцев центр линии, крылья линии, а также сателлит, порожденный эстремумом разностного потенциала [83]. Использование этой формулы привело к хорошему согласию с экспериментальными данными по поглощению для асимптотически запрещенного перехода

$$
\mathrm{Ca}\left(4 s^{21} S_{0}\right)+\mathrm{He}+\hbar \omega \rightarrow \mathrm{Ca}\left(4 s 3 d^{1} D_{2}\right)+\mathrm{He}
$$

в [84]. Наконец, в [85,86] получено равномерное квазиклассическое описание общего случая радиационного перехода между двумя экспоненциальными термами и экспоненциально зависящей от расстояния радиационной шириной. Переход к квазиклассике позволил, в частности, описать форму полосы излучения [87] в реакции

$$
\operatorname{Ar}\left(3 p^{5} 4 s^{3} P_{2}\right)+\mathrm{He} \rightarrow \operatorname{Ar}\left(3 p^{61} S_{0}\right)+\mathrm{He}+\hbar \omega
$$

при энергиях столкновений $\sim 100 \mathrm{~cm}^{-1}$.

Другое ограничение квазистатического приближения, использованного в разд. 1.7, связано с допущением, что состояние квазимолекулы $c 1\left({ }^{3} P_{2}\right)$ достаточно изолировано, так что можно пренебречь неадиабатическими переходами в другие состояния, и это вполне оправдано для экспериментальных данных, полученных в газовых ячейках. Но при переходе, например, к квазимолекулам, влючающим атомы главной подгруппы II группы элементов Таблицы Менделеева, особенно легкие щелочноземельные элементы, такое допущение уже не будет справедливым. Действительно, например, расщепление $\Delta \varepsilon\left({ }^{3} P_{2}-{ }^{3} P_{1}\right)=106(\mathrm{Ca}), 41(\mathrm{Mg}) \mathrm{cm}^{-1} \leq k T$, что должно быть учтено при вычислении спектральных профилей.

Далее при анализе процесса следует учитывать, что радиационные переходы происходят при всех межатомных расстояниях, при которых дипольный момент отличен от нуля, тогда как неадиабатический переход локализован в окрестности некоторого $R_{0}$, центра ограниченной области перехода. До достижения этой области в ходе столкновения и после ее прохождения система развивается адиабатически, так что спектр может быть описан на основе формулы (27). Разумеется, зависимости от расстояния как термов, так и дипольных моментов в этих двух областях будут различными. Другое важное отличие радиационных переходов внешних оболочек атома - они пропорциональны $\alpha^{3}$, и могут учитываться в рамках теории возмущений. Таким образом, влияние неадиабатичности на форму спектра может быть учтено следующим образом - сначала решается задача о неадиабатических переходах, а затем с полученными волновыми функциями, которые зависят от $R$, вычисляется спектр. Теория неадиабатических переходов при парных столкновениях атомов разработана достаточно подробно [88]. Единственное усложнение при вычислениях формы спектра связано с тем, что в отличие от теории столкновений теперь необходимо знание соответствующей волновой функции при всех $R$, а не только в асимптотических областях больших и малых $R$.

Как видно из рис. 2, 4, для термов конфигурации $n s n p$ характерны сравнительно слабая зависимость от расстояния при больших $R$ (термы почти горизонтальны) и быстрая перестройка в области средних $\sim(7-8) a_{0}$, т. е. ситуация, качественно соответствующая модели неадиабатических переходов Демкова [89]. В рамках этой модели профиль полосы определяется двумя параметрами [90]:

$$
\xi^{*}=\frac{\pi \Delta \varepsilon}{2 \alpha \sqrt{\frac{2 k T}{\mu}}}, \quad \Omega=\frac{2\left(\omega-\varepsilon_{0}\right)}{\Delta \varepsilon} .
$$


Первый из них - параметр Месси $(\mu-$ приведенная масса сталкивающихся атомов), который определяет вероятность перехода между состояниями, а второй энергия оптического перехода в единицах $\Delta \varepsilon-$ расщепления атомных термов ${ }^{3} P_{2,1}$, так что энергии атомных уровней в этой шкале $\Omega= \pm 1$. На рис. 10 приведен профиль полосы излучения для обсуждаемого случая, т.е. предполагается, что взаимодействуют два состояния, излучающее и метастабильное, причем изначально до столкновения заселено только метастабильное состояние. Спектральный профиль складывается из двух контуров. Это лоренцев контур с центром, который расположен на месте разрешенного перехода $\Omega=-1$, причем его интенсивность определяется вероятностью неадиабатического перехода из метастабильного в излучающее состояние. Второй контур, квазимолекулярный, который можно назвать сателлитом, формируется радиационными переходами при $R<R_{0}$ и $\omega \sim 1$ из состояния, которое при больших $R$ было метастабильно, но взаимодействие в области малых расстояний привело к частичной передаче дипольного момента излучающего состояния. В рамках модели Демкова для взаимодействия $V=V_{0} \exp (-\alpha R)$ такая перестройка радиационной ширины описывается формулой

$$
\Gamma_{q m}(R)=\Gamma_{a t} \sin ^{2} \theta(R), \theta(R)=\frac{1}{2} \operatorname{arctg}\left(\frac{V_{0}}{\Delta \varepsilon} e^{-\alpha R}\right)
$$

из работы [90], $\Gamma_{a t, q m}-$ радиационные ширины атомного диабатического и квазимолекулярного адиабатического состояний.

Согласно основной идее теории неадиабатических переходов, столкновения с $\xi^{*} \geq 1$ отвечают адиабатическому развитию системы, поэтому спектральные профили при таких условиях должны описываться формулами (28), (29). В качестве терма возбужденного состояния в этих формулах следует использовать расходящиеся адиабатические термы, а в качестве радиационной ширины - адиабатическую ширину (рис. 12, $b$, штриховая кривая). Количественно проследить зависимость максимума интенсивности сателлита и положения его центра $\Omega$ в зависимости от $\xi^{*}$ можно по рис. 11 . Видно, что при $\xi^{*}>1$ профиль сателлита удовлетворительно описывается формулами квазистатического приближения (27)-(29). Поскольку в общем случае сателлит образуется в ходе столкновения атомов, то в [90] он назван динамическим в отличие от сателлитов в крыльях линий, которые связаны с особенностями, например экстремумами, в зависимости термов $U^{*}, U_{0}$ от $R$.

Рассмотрим в качестве примера квазимолекулы $\mathrm{Ca}, \mathrm{Mg}-\mathrm{He}$. Интересные для радиационных процессов термы и радиационные ширины вычислены в одноконфигурационном приближении в подходе, обсужденном в разд. 1.2, 1.3, а также в [18]. Сравнение с многоконфигурационными расчетами в [91] показало, что в рассматриваемых случаях можно ограничиться одноконфигурационным расчетом. Незначительность отклонения

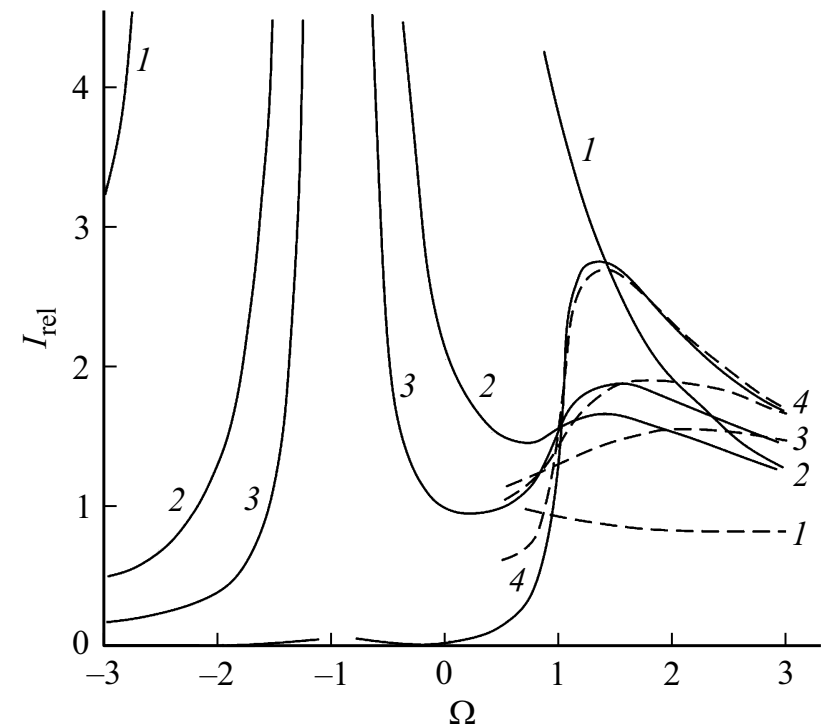

Рис. 10. Столкновительно индуцированный спектр, усредненный по параметрам удара и максвелловскому распределению скоростей, в случае первоначально заселенного состояния ${ }^{3} P_{2}(\Omega=1)$ [90] при различных значениях параметра Месси $\xi^{*}$. Сплошные линии - расчет в рамках модели Демкова, штриховые линии - расчет спектра в рамках адиабатического приближения на основе формулы (27): $\xi^{*}=0.1$ (1), 0.5 (2), $1(3), 5(4)$.

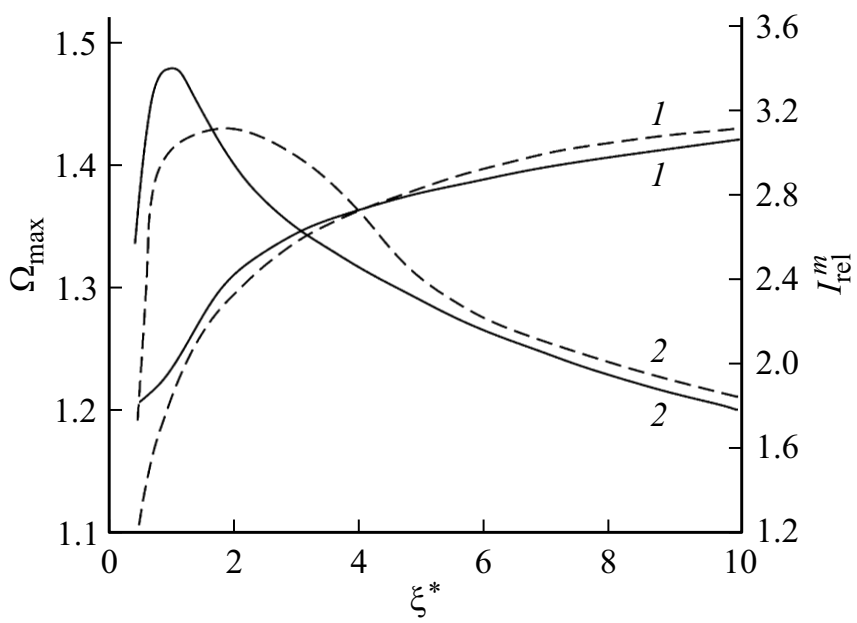

Рис. 11. Интенсивность столкновительно индуцированного максимума сателлита (1) и его положение (2) в зависимости от параметра Месси $\xi^{*}$ [90]. Сплошные кривые - расчет в рамках модели Демкова, штриховые кривые - расчет в рамках адиабатического приближения на основе формулы (27).

от правила интервалов Ланде -2.03 наводит на мысль ограничиться для описания процесса столкновения и спектрального профиля взаимодействием только двумя ближайшими диабатическими состояниями $\varphi_{2,3}$ (табл. 1) с проекцией момента $\Omega=1$. Поскольку диабатические термы параллельны, то процесс взаимодействия состояний может быть описан в рамках модели Демкова [90]. 

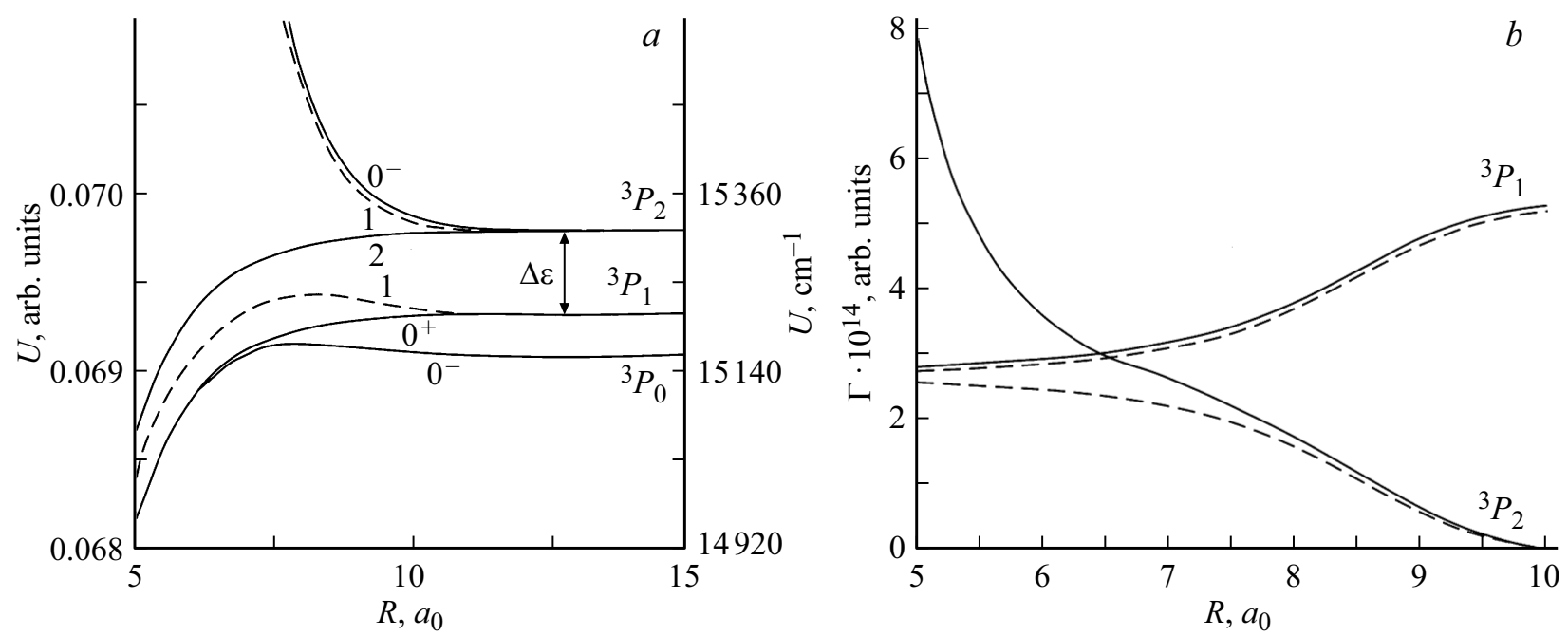

Рис. 12. Квазимолекулярные термы $(a)$ и ширины $(b) \mathrm{Ca}\left(4 s 4 p^{3} P_{j=0,1,2}\right)-$ Не. Сплошные кривые - одноконфигурационный расчет, штриховые - приближение двух состояний [92].

На рис. 12 из работы [92] штриховой кривой отмечены результаты расчетов термов и радиационных ширин в двухуровневом приближении в принятой модели. Как можно заключить из рис. 12 , для $R>6.5 a_{0}$ привлечение модели Демкова вполне оправдано, а квазистатическое приближение неприменимо, поскольку $\xi^{*}=0.8(\mathrm{Ca})$ и $\xi^{*}=0.22(\mathrm{Mg})$ при $T=1000 \mathrm{~K}$.

На рис. 13 приводится спектр, который образуется при столкновениях $\mathrm{Ca}\left(4^{3} P_{2}\right)+\mathrm{He}$ [92]. В результате столкновения проявляется разрешенный атомный переход $4^{3} P_{1}-4{ }^{1} S_{0}$ с красным крылом. Это крыло формируется переходами в области $R<R_{0}=8.8 a_{0}$, интенсивность которых пропорциональна вероятности неадиабатического перехода и зависит от частоты как $\left(\omega_{0}-\omega\right)^{-1}$. В фиолетовом крыле образуется сателлит, максимум которого сдвинут согласно расчету в рамках модели Демкова в фиолетовую область на $21 \mathrm{~cm}^{-1}$ от положения атомного уровня $\mathrm{Ca}\left(4^{3} P_{2}\right)$. Как следует из обсуждения выше, причина возникновения максимума связана с резким возрастанием от нуля до $\sim \Gamma_{a t} / 2$ радиационной ширины при прохождении области перестройки диабатических (атомных) функций в квазимолекулярные. Уточненный расчет, который учитывает взаимодействие с атомным состоянием $4^{1} P_{1}$ (сплошные кривые на рис. $12, b)$, увеличивает сдвиг еще на $75 \mathrm{~cm}^{-1}$. Расчет спектрального профиля в случае квазимолекулы $\mathrm{Mg}-\mathrm{He}$ в основных чертах воспроизводит уже описанные характеристики спектра для случая $\mathrm{Ca}-\mathrm{He}$, однако увеличение вероятности неадибатического перехода при уменьшении расщепления атомных уровней приводит к тому, что динамический сателлит не проявляется на фоне фиолетового крыла.

Важно отметить, что описание спектра при значениях параметра Месси $\xi^{*}<1$ возможно только с учетом взаимодействия состояний, а сложение спектров, вычисленных в квазистатическом приближении, интенсивно- сти которых даже „подправлены“ с учетом вероятности неадиабатического перехода, не воспроизводит форму результирующего спектра. Другая особенность - форма спектра зависит от того, какое атомное состояние было первоначально заселено, $4^{3} P_{1}$ или $4^{3} P_{2}[90,92,93]$.

\section{2. Квазимолекулы $\operatorname{Rg}\left(n p^{5}(n+1) s\right)-\mathbf{R g}^{\prime}\left({ }^{1} S_{0}\right)$}

\section{1. Введение}

Теоретические подходы, связанные с описанием радиационных переходов в несимметричных квазимолекулах инертных газов, включая и такие переходы, которые запрещены в пределе больших межатомных расстояний, уже обсуждались в работах обзорного характера $[94,95]$ Интересной для физики лазеров квазимолекуле $\mathrm{He}-\mathrm{Ne}$ в разных электронных состояниях посвящен обзор [96]. Экспериментальные результаты по радиационным переходам в несимметричных квазимолекулах собраны и прокомментированы в обзоре Герасимова [97]. Поэтому ниже рассматриваются отдельные работы, выполненные уже после публикации цитированных обзоров.

Выбор электронной конфигурации, указанной в заголовке, неслучаен. Как и выше, будет рассматриваться, по существу, двухчастичная задача - один электрон и одна дырка, т.е. по-прежнему 4 атомных состояния, но в обращенном порядке. (Разумеется, в случае конфигурации $\operatorname{Rg}\left(n p^{5} n^{\prime} l, l>0\right)$ такая аналогия пропадает.) Возникновение радиационной ширины состояния $\Omega=1$ также обусловлено взаимодействием трех состояний, термы которых переходят в атомные ${ }^{3} P_{2,1}$ и ${ }^{1} P_{1}$ (или в $s_{5,4,2}$ в обозначениях Пашена) при больших $R$.

Для $s$-электрона в атоме инертного газа эффективный заряд $\sim 1$, а для $p$-электронов $\sim 4$ [58]. Малость энергии связи возбужденного электрона обосновывает 
известную аналогию $\operatorname{Rg}\left(n p^{5}(n+1) s\right)$ с атомом щелочного металла, следующего в периодической системе за атомом $\operatorname{Rg}\left({ }^{1} S_{0}\right)$, и позволяет в полуэмпирической процедуре восстановления термов разд. 2.5 использовать надежно установленные термы квазимолекул щелочной металл-атом инертного газа. Также качественно понятно, что влияние обменного взаимодействия ионного остова с атомом буферного газа будет более существенным по сравнению с обменным взаимодействием с участием возбужденного электрона.

\section{2. Квазимолекулы легких инертных газов}

В работе [98] приводятся результаты первого многоконфигурационного расчета (MRD-CI) возбужденных состояний квазимолекулы $\mathrm{Ne}-\mathrm{He}$, которые при больших $R$ соответствуют состояниям $\mathrm{Ne}\left(2 p^{5} n s, n^{\prime} p, n^{\prime \prime} d\right)-\mathrm{He}\left(1 s^{2}\right), \quad n=3,4,5, \quad n^{\prime}=3,4$, $n^{\prime \prime}=3$ и $\mathrm{Ne}\left(2 p^{6}\right)-\mathrm{He}\left(1 s 2 s^{1,3} S\right)$. Расчеты подтвердили правильность заключения, сделанного в [99] для $n=3$ - область сильной связи состояний $1\left({ }^{3} P_{2}\right)$ и $1\left({ }^{3} P_{1}\right)$ при тепловых столкновениях не достигается, что позволяет, в частности, уверенно пренебречь вкладом радиационного тушения атомного состояния ${ }^{3} P_{2}$ по сравнению с неадиабатическим переходом в ближайшее состояние ${ }^{3} P_{1}$. Расчеты в [99] проводились в рамках метода псевдопотенциала и были, по существу, асимптотическими, справедливыми при $R>5 a_{0}$, тогда как в [98] удалось продвинуться в область $R>2.5 a_{0}$, где обнаружилась потенциальная яма $\left(R \sim 3 a_{0}\right)$ глубиной около $500 \mathrm{~cm}^{-1}$, превышающая энергию уровня ${ }^{3} P_{2}$ на $\sim 500 \mathrm{~cm}^{-1}$. Подобные особенности установлены и в расчетах с привлечением псевдопотенциала для термов, порожденных состояниями с $n=4,5$, что связано с поведением s-функции возбужденного электрона [96].

Расчет термов и дипольных моментов нижней возбужденной конфигурации $\operatorname{Ar}\left({ }^{3} p^{5} 4 s^{1,3} P\right)-\mathrm{Ne}$ в рамках метода MRD-CI с релятивистскими потенциалами для остова (RECP) [100] позволил сделать интересное сопоставление с результатами вычисленией с использованием псевдопотенциала [101] и модельного потенциала [102]. Для состояния $1 s_{5}$ расчет [100] показал наличие потенциальной ямы глубиной $793 \mathrm{~cm}^{-1}$ при $R_{m}=7.796 a_{0}$, расчет с привлечением псевдопотенциала дал $40 \mathrm{~cm}^{-1}$, $R_{m}=8.5 a_{0}$, а расчет в рамках модельного потенциала не выявил минимума в потенциальной кривой. Далее по результатам [100] время жизни состояния $v=0$ в яме составляет $8.3 \mu \mathrm{s}$, тогда как по данным [101] величина $1 / \Gamma\left\lfloor 1\left(s_{5}\right), R \approx 7.3 a_{0}\right\rfloor=2 \mathrm{~ns}$. Видимо, справедливо считать, что большинство результатов, полученных в аналитическом подходе с использованием псевдопотенциала и приведенных в [94-96], правильно качественно и полуколичественно отражают существо многочисленных процессов с участием возбужденных атомов инертных газов, в частности, причину возникновения дипольного момента перехода $1\left({ }^{3} P_{2}\right) \leftrightarrow 0^{+}\left({ }^{1} S_{0}\right)$. Что касается надежных количественных результатов для отдельных

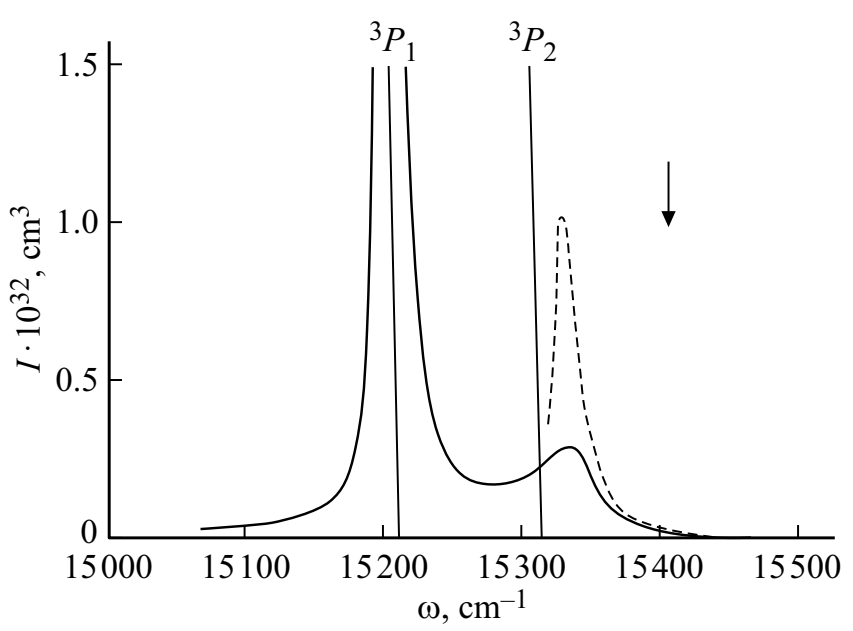

Рис. 13. Квазимолекула Са-Не. Зависимость спектральной интенсивности от частоты [92]. Слошными вертикальными прямыми отмечены положения атомных уровней ${ }^{3} P_{1,2}$. Штриховая кривая - квазимолекулярный переход $(\Omega=1$, $\left.4 s 4 p^{3} P_{2}\right) \rightarrow\left(\Omega=0^{+}, 4 s^{21} S_{0}\right)$ в квазистатическом приближении (28). Вертикальной стрелкой отмечен сдвиг максимума при учете взаимодействия с состоянием ${ }^{1} P_{1}$. Сплошная линия - расчет в рамках модели Демкова при начальном заселении состояния ${ }^{3} P_{2}$.

квазимолекул, то здесь неоспоримо преимущество различных вариантов расчетов $a b$ initio.

\section{3. Квазимолекулы тяжелых инертных газов}

Вычисление термов несимметричных квазимолекул, образованных атомами тяжелых инертных газов, в полуэмпирическом подходе, аналогичном описанному в разд. $1.2,1.5$, уже подробно комментировалось в $[95,94]$. В табл. 10 приведены вычисленные в этом подходе параметры потенциальных ям для некоторых квазимолекул [103]. Как видно, для тяжелых квазимолекул характерно наличие потенциальных ям, существенно превышающих $k T$. В полуэмпирическом подходе можно вычислять и ширины радиационных переходов аналогично тому, как это описано в разд. 1.6. Здесь уместно сослаться на замечание в конце предыдущего раздела. В работе [104] выполнен расчет состояний $\mathrm{Xe}\left({ }^{3} P_{1,2}\right)-\mathrm{Kr}$ в подходе $a b$ initio с учетом релятивистких поправок и корреляции электронов. Несмотря на совпадение структуры термов и положения минимумов в области средних расстояний $R<10 a_{0}$, значения минимумов потенциалов расходятся (табл. 10).

Для квазимолекул инертных газов основное отличие от результатов разд. 1 состоит в том, что определяющим взаимодействием для перестройки атомных диабатических функций теперь будут зависящие от расстояния расщепления $V_{\Sigma, \Pi}$ термов молекулярного ионного остова, которое в области средних расстояний превосходит взаимодействие возбужденного электрона с остовом. Понятно, что перестройка влияет как на формирование 
Таблица 10. Равновесные межъядерные расстояния и глубины $U_{m}$ потенциальных ям для состояния $1\left(s_{5}\right)$ квазимолекул $\mathrm{Xe}^{*}+\mathrm{Ar}, \mathrm{Xe}^{*}+\mathrm{Kr}$ и $\mathrm{Kr}^{*}+\mathrm{Ar}[103]$

\begin{tabular}{c|c|c|c}
\hline Параметр & $\mathrm{Xe}^{*}+\mathrm{Ar}$ & $\mathrm{Xe}^{*}+\mathrm{Kr}$ & $\mathrm{Kr}^{*}+\mathrm{Ar}$ \\
\hline$R_{m}, a_{0}$ & 7.75 & 6.50 & 6.00 \\
& $7.75^{*}$ & $6.15^{*}$ & $6.05^{*}$ \\
& & $5.89^{* *}$ & \\
$U_{m}, \mathrm{~cm}^{-1}$ & 438.9 & 1119 & 526.7 \\
& $307.3^{*}$ & $1119^{*}$ & $855.9^{*}$ \\
& & $51^{* *}$ &
\end{tabular}

Примечание. * —экспериментальные данные [105], ** — расчет [104].

потенциальных ям, так и на формирование максимума дипольного момента перехода в основное состояние, и как следствие, на времена жизни колебательных состояний $\tau_{v}$. Поэтому величина $\tau_{v}$ оказывается весьма чувствительной к взаимному расположению потенциальной ямы и зависимости дипольного момента от $R$ в этой области.

В работе [106] вычисляется $\tau_{v}$ для эксимеров $\mathrm{XeKr}$, $\mathrm{XeAr}, \mathrm{KrAr}$ в состоянии $1\left({ }^{3} P_{2}\right)$. Для рассмотренных пар $R_{m} \sim(6-7) a_{0}$, а положение максимума радиационной ширины либо совпадает, либо несколько меньше $R_{m}$, поэтому значение интеграла $\tau_{v}^{-1} \sim\langle v|\Gamma| v\rangle$ определяется областью $R$, в которой ширина быстро убывает с увеличением расстояния. Как результат $\tau_{v}$ увеличивается с ростом колебательного возбуждения, тогда как в симметричном случае $\mathrm{Ar}_{2}$ [107] зависимость противоположная, поскольку $R_{m}$ для симметричного случая меньше и находится слева от положения максимума ширины. Несмотря на то, что время жизни - интегральная характеристика, она оказалась чувствительной к деталям взаимодействия атомов в состоянии $1\left({ }^{3} P_{2}\right)$ или $\mathrm{A} 1_{u}$.

В работе [108] изучалось, в частности, влияние различия в потенциалах квазимолекулярных состояний и радиационной ширины на усредненный спектральный профиль. Привлечение аналитического подхода позволило сделать следующее наблюдение. При анализе профилей спектральных линий посредством варьирования зависимостей потенциалов и ширин нередко добиваются хорошего согласия экспериментальных и расчетных данных. Но как продемонстрировано в [108], для асимптотически запрещенных переходов при таком подходе следует соблюдать определенную осторожность. Отличающиеся наборы термов основного и возбужденного состояний могут порождать похожие профили, но при этом благодаря сильной зависимости радиационной ширины от $R$ дают отличающиеся на порядок константы радиационного тушения. В таких случаях аналитические подходы к определению термов и ширин могут помочь избежать ошибок при определении областей расстояний, переходы в которых дают определяющий вклад в интегральные и дифференциальные характеристики радиационных переходов.

\section{4. На пути к полному опыту}

Несмотря на то, что привлечение экспериментальных данных в вышеизложенном было скромным и несистематичным, очевидно, что обсуждавшиеся расчеты были ориентированы на эксперименты в условиях газовой ячейки. Хотя подавляющее количество экспериментальных данных по поглощению или излучению получено именно в таких условиях, двойное усреднение (например, по параметрам удара в полуклассическом приближении и максвелловскому распределению), которое приходится выполнять в расчетах для последующего сравнения, маскирует влияние деталей поведения потенциальных кривых и дипольных моментов перехода. Поэтому изучение дифференциальных сечений низкоэнергетического рассеяния с регистрацией оптических спектров и анализом поляризации могло бы поспособствовать пониманию радиационных процессов при взаимодействии сталкивающихся атомов. Полезным здесь может оказаться равномерный квазиклассический подход, который не использует известные модели атомных столкновений со специфической зависимостью квазимолекулярых термов от расстояния и который включает явным образом зависимость от орбитального момента. Последнее обстоятельство важно, поскольку увеличение орбитального момента сопровождается качественным изменением картины формирования оптического спектра.

Примеры расчетов представлены в работах [87], где равномерная квазиклассическая формула использована, в частности, для описания излучения при дифференциальном рассеянии $\operatorname{Ar}\left({ }^{3} P_{2}\right)+$ Не при энергиях столкновений 100, 200 и $1000 \mathrm{~cm}^{-1}$, и [109], в которой выполнен первый расчет однофотонного поглощения при столкновениях $\mathrm{He}\left(1^{1} S\right)+\mathrm{Ne}+\hbar \omega \rightarrow \mathrm{He}\left(2^{1} S\right)+\mathrm{Ne}$ с энергией $219 \mathrm{~cm}^{-1}$. В последней работе установлена зависимость полных и дифференциальных сечений от взаимной ориентации векторов поляризации падающего излучения и начальной относительной скорости, а также частоты отстройки от атомного перехода. Было бы интересно проделать подобный расчет и для селективного получения атомов $\operatorname{Rg}\left({ }^{3} P_{2}\right)$.

\section{3. Заключение}

Описанные выше аналитические подходы позволили обсудить различные радиационные процессы при парных столкновениях с участием довольно широкого набора атомов. Разумеется, такие подходы не могут претендовать на точность, достижимую в подходах $a b$ initio, но они открывают возможность для понимания общих закономерностей различных радиационных процессов при столкновениях. Для приложений такие подходы также могут оказаться полезными, поскольку позволяют сравнительно просто оценить вклады различных процессов в заселение и распад метастабильных состояний. Перспективным представляется развитие аналитических 
подходов для анализа дифференциальных сечений, в частности, с участием поляризованных атомов. Определенные шаги в этом направлении уже сделаны в работах $[110,111]$.

\section{Благодарности}

Особая благодарность автора за сотрудничество его бывшим аспирантам, а ныне коллегам, А.К. Беляеву, Е.Н. Бичуцкой, А.Л. Загребину, Н.А. Павловской, Е.А. Чеснокову, а О.С. Алексеевой и М.Г. Ледневу еще и за большую помощь при оформлении работы.

\section{Финансирование работы}

Исследование выполнено при финансовой поддержке РФФИ в рамках научного проекта „Экспансия“ № 2012-50260/20.

\section{Конфликт интересов}

Автор заявляет, что у него нет конфликта интересов.

\section{Список литературы}

[1] Allard N., Kielkopf J. // Rev. Mod. Phys. 1982. V. 54(4). P. 1103.

[2] Garstang R.H. // Atomic and Molecular Processes / Ed. by Bates D.R. Academic Press, 1962. 576 p. Перевод: Гарстанг P. // Атомные и молекулярные процессы / Под ред. Бибермана Л.И., Фабриканта В.А. М.: Мир, 1964. С. 9.

[3] Девдариани А.З., Себякин Ю.Н. // ЖЭТФ. 1989. Т. 96. В. 6. C. 1997; Devdariani A.Z., Sebyakin Yu.N. // Sov. Phys. JETP. 1989. V. 96. N 7. P. 1127.

[4] Дубов В.С., Никоненко С.Г. // ЖЭТФ. 1991. Т. 99. С. 735; Dubov V.S., Nikonenko S.G. // JETP. 1991. V. 72. N 3. P. 407.

[5] Behmenburg W., Makonnen A., Kaiser A., Rebentrost F., Staemler V., Jungen M., Peach $G$., Devdariani A., Tserkovnyi S., Zagrebin A., Czuchaj E. // J. Phys. B. 1996. V. 29. P. 3891.

[6] Gallagher A. // Atomic, Molecular, \& Optical Physics / Ed. by Gordon W.F., Drake A. Springer, 2006. P. 220.

[7] Allison D.C., Brown J.C., Dalgarno A. // Proc. Phys. Soc. 1966. V. 89. P. 41.

[8] Devdariani A., Leboucher-Dalimier E., Sauvan P., Angelo P. // Phys. Rev. A. 2002. V. 66. P. 052703.

[9] Devdariani A., Dalimier E., Sauvan P. // Int. J. Spectr. 2010. V. 2010. Article ID 812471. Режим доступа: http: // www.hindawi.com/journals/ijs/2010/812471.html. doi: $10.1155 / 2010 / 812471$

[10] Koperski J. // Phys. Rep. 2002. V. 369. P. 177.

[11] Девдариани А.З., Загребин А.Л. // Опт. и спектр. 1985. T. 58. B. 6. C. 1223; Devdariani A.Z., Zagrebin A.L. // Opt. Spectrosc. 1985. V. 58. N 6. P. 752.

[12] Nikitin E.E., Umanskii S.Ya. Theory of Slow Atomic Collisions. Springer, 1984. doi: 10.1007/978-3-642-82045-8

[13] Смирнов Б.М. Асимптотические методы в теории атомных столкновений. 1973. 295 с.

[14] Загребин А.Л., Леднев С.И., Церковный С.И. // Опт. и спектр. 1993. Т. 74. В. 1. С. 24.
[15] Загребин А.Л., Леднев М.Г. // Опт. и спектр. 1985. Т. 79. B. 6. C. 912.

[16] Загребин А.Л., Леднев М.Г. // Опт. и спектр. 1995. Т. 78. № 2. C. 183.

[17] Kurosawa T., Ohmory K., Chiba H., Okunishi M., Ueda K., Sato Y., Devdariani A.Z., Nikitin E.E. // J. Chem. Phys. 1998. V. 108. N 19. P. 8101.

[18] Загребин А.Л., Леднев М.Г. // Опт. и спектр. 1992. Т. 72. B. 3. C. 535.

[19] Lurio A. // Phys. Rev. 1965. V. 140. P. A1505.

[20] Krykov N.A., Penkin N.P., Red'ko T.P. // Opt. Spectrosc. 1981. V. 51. P. 756.

[21] Czuchaj E., Krośnicki M., Stoll E. // Chem. Phys. 1991. V. 263. P. 7.

[22] Fermi E. // Nuovo Cimento. 1934. V. 11. P. 157.

[23] Загребин А.Л., Леднев С.И., Церковный С.И. // Опт. и спектр. 1993. Т. 74. В. 1. С. 24.

[24] Загребин А.Л., Церковный С.И. // Хим. физика. 1990. Т. 9. № 6. C. 727.

[25] Иванов Г.К. // Теор. эксп. химия. 1978. Т. 14. № 5. С. 610; Ivanov G.K. // Theor. Exp. Chem. 1978. V. 14. N 5. P. 472.

[26] Загребин А.Л., Павловская Н.А. // Хим. физика. 1988. Т. 7. C. 435.

[27] Загребин А.Л., Церковный С.И. // Хим. физика. 1990. Т. 9. № 6. C. 727.

[28] Груздев П.Ф., Шерстюк А.И. // Опт. и спектр. 1976. Т. 40. B. 4. C. 617.

[29] Van Zee R.D., Blankespoor S.C., Zweier T.S. // Chem. Phys. Lett. 1989. V. 158. P. 306.

[30] Дашевская Е.И., Девдариани А.З., Загребин А.Л. // Химия плазмы. М.: Энергоатомиздат. 1987. № 14. С. 127; Dashevskaya E.I., Devdariani A.Z., Zagrebin A.L. // Reviews of Plasma Chemistry. Kluwer. 1991. V. 1.

[31] Девдариани А.З., Загребин А.Л., Леднев М.Г. // Хим. физика. 1998. Т. 17 № 6. C. 70; Devdariani A.Z., Zagrebin A.L., Lednev M.G. // Chem. Phys. Reports. 1998. V. 17. N 6. P. 1107.

[32] Девдариани А.З., Загребин А.Л., Леднев М.Г., Алексеев А.Б., Либерман Х.-П., Буенкер Р.Дж. // Опт. и спектр. 2001. Т. 91. С. 891; Devdariani A.Z., Zagrebin A.L., Lednev M.G., Alekseyev A.B., Liebermann H.P., Buenker R.J. // Opt. Spectrosc. 2001. V. 91. N 6. P. 833.

[33] Загребин А.Л., Церковный С.И. // Хим. физика. 1990. Т. 9. № 6. С. 727 .

[34] Загребин А.Л., Церковный С.И. // Хим. физика. 1991. Т. 10. № 5. C. 595.

[35] Behmenburg W., Makkonen A., Kaiser A., Rebentrost F., Staemmler V., Jungen $M$, Peach G., Devdariani A., Tserkovnyi S., Zagrebin A., Czuchaj E. // J. Phys. B. 1996. V. 29. P. 3891.

[36] Buenker R.J., Peyerimhoff S.D. // Theor. Chim. Acta. 1974. V. 35. P. 33.

[37] Buenker R.J., Peyerimhoff S.D. // Theor. Chim. Acta. 1975. V. 39. P. 17.

[38] Buenker R.J., Peyerimhoff S.D., Butscher W. // Mol. Phys. 1978. V. 35. P. 771.

[39] Ermer W.C., Ross., Cristiansen P.A. // Adv. Quant. Chem. 1988. V. 19. P. 139.

[40] Alekseyev A.B., Libermann H.-P., Buenker R.J., Hirsh G., Li Y. // J. Chem. Phys. 1994. V. 100. P. 8956.

[41] Buenker R.J., Alekseyev A.B., Libermann H.-P., Zingott R., Hirsh G. // J. Chem. Phys. 1998. V. 108. P. 3400. 
[42] Czuchaj E., Stoll H., Preuss H. // J. Phys. B. 1987. V. 20. P. 1487.

[43] Yamanauchi K., Isogai S., Okunishi M., Tsuchiya S. // J. Chem. Phys. 1988. V. 88. P. 306.

[44] Van Zee R.D., Blankespour S.C., Zwier T.S. // Chem. Phys. Lett. 1989. V. 158. P. 306.

[45] Duval M.-C., Jouvet C., Soep B. // Chem. Phys. Lett. 1985. V. 119. P. 317.

[46] Загребин А.Л., Леднев М.Г. // Опт. и спектр. 1995. Т. 79. № 6. C. 912.

[47] Загребин А.Л., Леднев М.Г. // Опт. и спектр. 1997. Т. 83. № 2. C. 212.; Zagrebin A.L., Lednev M.G. // Opt. Spectrosc. 1997. V. 83. N 2. P. 196.

[48] Krykov N.A., Penkin N.A., Red'ko T.P. // Opt. Spectrosc. 1989. V. 66. N 6. P. 1235.

[49] Callear A.B, Du K. // Chem. Phys. 1987. V. 113. N 1. P. 73.

[50] Amano K., Ohmori K., Kurosawa T., Chiba H., Okunishi M., Ueda K., Sato Y., Devdariani A.Z., Nikitin E.E. // J. Chem. Phys. 1998. V. 108. N 19. P. 8110.

[51] Alekseeva O.S., Devdariani A.Z., Lednev M.G, Zagrebin A.L. // Chem. Phys. Lett. 2013. V. 572. P. 141.

[52] Загребин А.Л., Леднев М.Г. // Опт. и спектр. 1995. Т. 78. № 5. C. 758.

[53] Остроухова И.И., Смирнов Б.М., Шляпников Т.В. // ЖЭТФ. 1977. Т. 73. № 1. С. 166.

[54] Беляев А.К., Девдариани А.З., Себякин Ю.Н. // Опт. и спектр. 1985. Т. 59. В. 3. С. 505.

[55] Девдариани А.З., Себякин Ю.Н. // ЖПС. 1985. Т. 42. № 1. C. 29.; Devdariani A.Z., Sebyakin Y.N. // J. Appl. Spectr. 1985. V. 42. N 1. P. 22.

[56] Alekseeva O.S., Devdariani A.Z., Lednev M.G, Zagrebin A.L. // J. Phys. Conf. Series. 2012. V. 397. P. 012031

[57] Ohmory K., Kurosawa T., Chiba H., Okunishi M., Sato Y., Devdariani A.Z., Nikitin E.E. // Chem. Phys. Lett. 1999. V. 315. P. 411.

[58] Собельман И.И. Введение в теорию атомных спектров. М.: Наука, 1977. 640 c.; Sobel'man I.I. An Introduction to the Theory of Atomic Spectra. Elsevier, 1972. 608 p.

[59] Devdariani A.Z., Kryukov N.A., Zagrebin A.L., Lednev M.G., Timofeev N.A. // JQSRT. 2020. V. 248. P. 106951.

[60] Devdariani A.Z., Klucharev A.N. // Opt. Spectrosc. 1977. V. 42. N 6. P. 694.

[61] Амусья М.Я. Атомный фотоэффект. М.: Наука, 1987.

[62] Devdariani A., Kereselidze T.M., Noselidze I.L., Dalimier E., Sauvan P., Angelo P., Schott R. // Phys. Rev. A. 2005. V. 71. N 2. P. 022512 .

[63] Алексеева О.С., Девдариани А.З., Загребин А.Л., Леднев М.Г. // Хим. физика. 2011. Т. 30. № 11. С. 73; Rus. J. Phys. Chem. B. 2011. V. 5. N 6. P. 946.

[64] Алексеева О.С., Девдариани А.З., Леднев М.Г., Загребин А.Л. // Хим. физика. Т. 34. № 8. С. 9; Rus. J. Phys. Chem. B. 2015. V. 9. N 4. P. 515

[65] Yamanouchi K., Isogai S., Okunishi M., Tsuchiya S. // J. Chem. Phys. 1988. V. 88. N 1. P. 205.

[66] Koperski J., Lukomski M., Czajkowski M. // Spectrochim. Acta. A. 2002. V. 58. P. 2709.

[67] Радииг А.А., Смирнов Б.М. Справочник по атомной и молекулярной физике. М.: Атомиздат, 1980. 240 с.; Radzig A.A., Smirnov B.M. Reference data on atoms, molecules and ions. Berlin: Springer-Verlag, 1985.
[68] Kramers H.A., ter Haar D. // Bull. Astr. Inst. Netherlands. 1946. V. 10. P. 137.

[69] Bates D.R. // Mon. Not. R. Astr. Soc. 1952. V. 112. P. 40.

[70] Jablonski A. // Proc. Int. Conf. on Opt. Pumping and Atomic Line Shapes. Warsaw, 1968. P. 323.

[71] Szudy J., Baylis W. // Phys. Rep. 1996. V. 266. P. 130.

[72] Яковленко С.И. Радиационно-столкновительные явления. М.: Энергоатомиздат, 1984. 209 с.

[73] Девдариани А.3. // Опт. и спектр. 2015. Т. 119. № 3. С. 356.; Devdariani A.Z. // Opt. Spectrosc. 2015. V. 119. N 3. P. 333.

[74] Загребин А.Л., Леднев М.Г. // Письма в ЖТФ. 1989. Т. 15. № 24. C. 11.

[75] Загребин А.Л., Леднев М.Г. // Опт. и спектр. 1998. Т. 85. № 2. C. 200; Zagrebin A.L., Lednev M.G. // Opt. Spectrosc. 1998. T. 85. N 2. C. 181.

[76] Sato Y., Nakamura T., Okunishi M, Ohmori K., Chiba H., Ueda K. // Phys. Rev. A. 1996. V. 53. P. 867.

[77] Загребин А.Л., Леднев М.Г. // Опт. и спектр. 1999. Т. 87. № 6. C. 893.; Zagrebin A.L., Lednev M.G. // Opt. Spectrosc . 1999. V. 87. N 6. P. 812.

[78] Русов Д.В., Себякин Ю.Н. // Опт. и спектр. 1999. Т. 87. № 1. C. 22; Rusov D.V., Sbyakin Yu.N. // Opt. Spectrosc. 1999. V. 87. N 1. P. 16.

[79] Devdariani A.Z., Ostrovskii V.N., Niehaus A. // J. Phys. B. 1985. V. 18. P. L161.

[80] Szudy J., Baylis W.E. // JQSRT. 1975. V. 15. P. 641.

[81] Devdariani A., Sauvan P., Leboucher-Dalimier E., Angelo P., Gauthier P. // Rapport cienifique. 1996. Lab. L'Utilisation Laser Intenses Ecole Polytechnique NTIS: B97-170963. P. 115.

[82] Devdariani A., Bichoutskaia E., Tchesnokov E., Bichoutskaia T., Crothers D.S.F., Leboucher-Dalimier E., Sauvan P., Angelo P. // J. Phys. B. 2002. V. 35. P. 2469.

[83] Девдариани А.3. // Опт. и спектр. 1999. Т. 86. С. 954; Devdariani A.Z. // Opt. Spectrosc. 1999. V. 86. P. 853.

[84] Bichoutskaia E., Devdariani A., Ohmori K., Misaki O., Ueda K., Sato Y. // J. Phys. B. 2001. V. 34. P. 2301.

[85] Девдариани А.З. // ЖЭТФ. 1989. Т. 96. С. $472 . ;$ Devdariani A.Z. // J. Exp. Theor. Phys. 1989. V. 98. P. 486.

[86] Девдариани А.З., Чесноков Е.А. // Хим. физика. 1998. Т. 17. № 6. C. 57.; Devdariani A.Z., Chesnokov E.A. // Chem. Phys. Reports. 1998. V. 17. P. 10.

[87] Девдариани А.З., Загребин А.Л., Ребентрост Ф., Церковный С.И. Чесноков Е.А. // ЖЭТФ. 2002. Т. 122. № 3. С. 481.

[88] Никитин Е.Е, Смирнов Б.М. Медленные атомные столкновения. М.: Энергоатомиздат, 1990. 256 с.

[89] Демков Ю.Н. // ЖЭТФ. 1963. Т. 45. С. 195; Demkov Yu.N. // Sov. Phys. JETP. 1964. V. 18. P. 138.

[90] Бичуцкая Е.Н., Девдариани А.З., Себякин Ю.Н. // Опт. и спектр. 1998. Т. 85. № 1. С. 11; Bichoutskaya E.N., Devdariani A.Z., Sebyakin Yu.N. // Opt. Spectrosc. 1999. V. 85. № 1. P. 7.

[91] Загребин А.Л., Церковный С.И. // Опт. и спектр. 1993. T. 72. B. 2. C. 276.

[92] Бичуикая Е.Н., Девдариани А.З., Загребин А.Л., Себякин Ю.Н. // Опт. и спектр. 1999. Т. 87. № 2. C. 213; Bichoutskaia E.N., Devdarainai A.Z., Zagrebin A.L., Sebyakin Yu.N. // Opt. Spectr. 1999. V. 87. N 2. P. 197.

[93] Devdariani A.Z. // Spectral Line Shapes. 1999. V. 10. 14th ICSLS ed. R.M. Herman: AIP Conf. Proc. 467. P. 325.

[94] Devdariani A.Z., Zagrebin A.L., Blagoev K.B. // Ann. Phys. Fr. 1989. V. 14. N 5. P. 467. 
[95] Девдариани А.З., Загребин А.Л. // Химия плазмы / Под ред. Смирнова Б.М. Сб. статей. В. 15. М.: Энергоатомиздат, 1989. C. 44.

[96] Devdariani A.Z., Zagrebin A.L., Blagoev K.B. // Ann. Phys. Fr. 1992. V. 17. N 5. P. 365.

[97] Герасимов Г.Н. // УФН. 2004. Т. 174. С. 155.

[98] Buenker R.J., Liebermann H.-P., Devdariani A.Z. // J. Phys. Chem. 2007. V. 111. N 7. P. 1307.

[99] Девдариани А.З., Загребин А.Л. // Опт. и спектр. 1985. T. 59. B. 2. C. 256; Devdariani A.Z., Zagebin A.L. // Opt. Spectrosc. 1985. V. 59. N 2. P. 155.

[100] Petsalakis I.D., Buenker R.J., Lieberman H.-P., Alekseyev A.B., Devdariani A.Z., Theodorakopoulos G. // J. Chem. Phys. 2000. V. 113. N 14. P. 5812.

[101] Загребин А.Л., Павловская Н.А. // Опт. и спектр. 1986. T. 62. B. 2. C. 264.

[102] Spiegelmann F., Gadea F.X., Castex M.C. // Chem. Phys. 1990. V. 145. P. 173.

[103] Загребин А.Л., Павловская Н.А. // Опт. и спектр. 1990. T. 69. B. 3. C. 534.

[104] Jansik B., Schimmelpfennig B., Ågren H. // Phys. Rev. A. 2003. V. 67. P. 042501.

[105] Nowak J., Fricke J. // J. Phys. B. 1985. V. 18. N 7. P. 1355.

[106] Zagrebin A.L., Tserkovnyi S.I. // Chem. Phys. Lett. 1995. V. 239. P. 136.

[107] Madey A.A., Herman P.R., Stoicheff B.P. // Phys. Rev. Lett. 1985. V. 57. P. 1574.

[108] Devdariani A., Chesnokov E., Zagrebin A., Lednev M.G., Petsalakis I.D., Theodorakopoulos G., Liebermann H.-P., Buenker R.J. // Chem. Phys. 2006. V. 330. P. 101.

[109] Devdariani A., Chesnokov E., Rebentrost F. // Chem. Phys. 2015. V. 462. P. 12.

[110] Чесноков Е.A // ЖЭТФ. 2005. Т. 128. № 5. С. 883.

[111] Загребин А.Л. // ЖЭТФ. 1990. Т. 70. №1. С. 7 114; Zagrebin A.L. // Sov. Phys. JETP. 1990. V. 70. P. 64. 